\title{
10. NSW HOSPITALS
}

\section{Onset and augmentation of labour in selected hospitals}

Table 115 gives onset or augmentation of labour for individual hospitals where the number of reported deliveries exceeded 200 in 2003, totals for all hospitals within each health area and the NSW total.

\section{TABLE 115}

CONFINEMENTS BY ONSET AND AUGMENTATION OF LABOUR AND HOSPITAL, NSW 2003\#

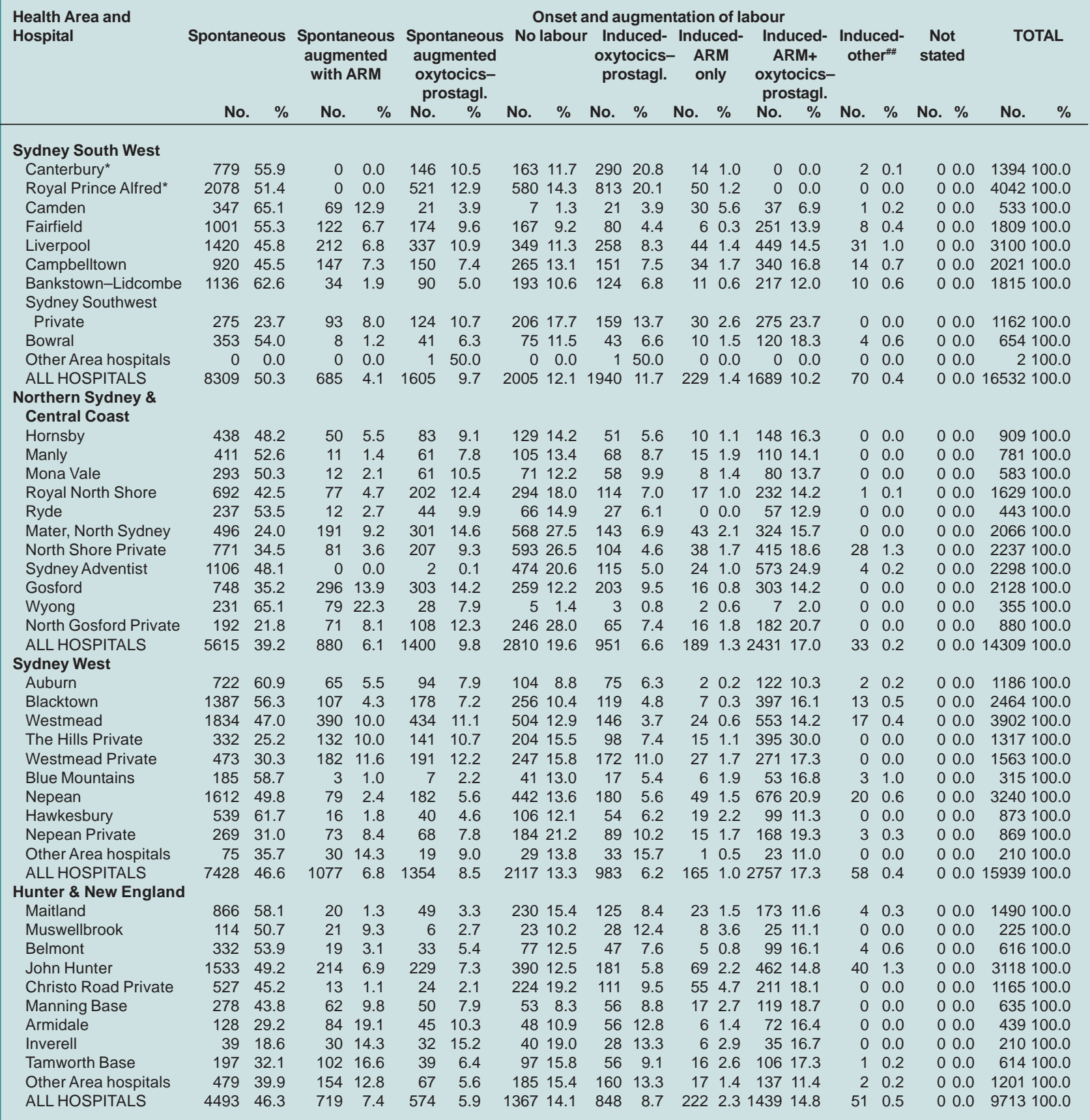


TABLE 115 (continued)

CONFINEMENTS BY ONSET AND AUGMENTATION OF LABOUR AND HOSPITAL, NSW $2003^{\#}$

Health Area and

Hospital
Onset and augmentation of labour

Spontaneous Spontaneous Spontaneous Nolabour Induced- Induced- Induced- Induced- Not TOTAL

$$
\text { augmented augmented oxytocics- ARM ARM+ other"\# stated }
$$$$
\text { with ARM oxytocics- prostagl. only oxytocics- }
$$

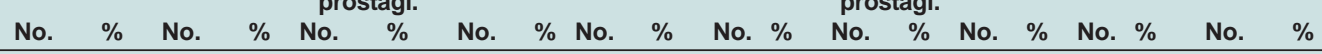

South Eastern Sydney \&

Illawarra

Shoalhaven

Wollongong

Shellharbour

Illawarra Private

Royal Hospital

for Women

St. George

Sutherland

$419 \quad 58.6$

$654 \quad 33.8$

$97 \quad 45.1$

$238 \quad 23.8$

198254.0

$1375 \quad 61.7$

$\begin{array}{ll}407 & 52.4\end{array}$

$\begin{array}{ll}407 & 52.4 \\ 274 & 23.7\end{array}$

$105 \quad 15.3$

Kareena Private

$509 \quad 32.2$

St. George Private

$\begin{array}{ll}509 & 32.2 \\ 443 & 26.0\end{array}$

$\begin{array}{lrr}\text { Prince of Wales Private } & 443 & 26.0 \\ \text { Other Area hospitals } & 70 & 48.3\end{array}$

ALL HOSPITALS

$6573 \quad 41.6$

North Coast

Grafton Base

Lismore Base

Murwillumbah

Tweed Heads

Coffs Harbour Base

Kempsey

Port Macquarie Base

$573 \quad 41.6$

$\begin{array}{rrrr}25 & 3.5 & 27 & 3.8 \\ 387 & 20.0 & 241 & 12.4\end{array}$

$\begin{array}{lllr}387 & 20.0 & 241 & 12.4\end{array}$

$\begin{array}{llll}39 & 18.1 & 20 & 9.3\end{array}$

$\begin{array}{llll}97 & 9.7 & 97 & 9.7\end{array}$

$\begin{array}{llll}33 & 0.9 & 238 & 6.5\end{array}$

$\begin{array}{llll}63 & 2.8 & 150 & 6.7\end{array}$

$\begin{array}{llll}19 & 2.4 & 62 & 8.0\end{array}$

$\begin{array}{llll}91 & 7.9 & 174 & 15.1\end{array}$

$\begin{array}{llll}38 & 5.5 & 82 & 12.0\end{array}$

$\begin{array}{llll}138 & 8.7 & 188 & 11.9\end{array}$

$\begin{array}{llll}233 & 13.7 & 193 & 11.3\end{array}$

$\begin{array}{rrrr}2 & 1.4 & 6 & 4.1\end{array}$

$\begin{array}{llll}1165 & 7.4 & 1478 & 9.3\end{array}$

$\begin{array}{llllll}171 & 42.4 & 39 & 9.7 & 23 & 5.7\end{array}$

$546 \quad 46.0$

$\begin{array}{ll}149 & 40.2\end{array}$

$352 \quad 41.5$

$292 \quad 39.8$

$\begin{array}{ll}114 & 41.2\end{array}$

$128 \quad 10.8$

$\begin{array}{ll}87 & 7.3\end{array}$

$\begin{array}{llll}56 & 15.1 & 38 & 10.2\end{array}$

$\begin{array}{llll}96 & 11.3 & 61 & 7.2\end{array}$

$\begin{array}{llll}76 & 10.4 & 48 & 6.5\end{array}$

$\begin{array}{llll}56 & 20.2 & 17 & 6.1\end{array}$

$\begin{array}{llll}122 & 17.1 & 63 & 8.8\end{array}$

$\begin{array}{llll}70 & 14.3 & 24 & 4.9\end{array}$

64312.8

$361 \quad 7.2$

ALL HOSPITALS

Greater Western

Dubbo Base

Mudgee

Bathurst Base

Orange Base

Broken Hill Base

Other Area hospitals

$291 \quad 59.3$

$2170 \quad 43.2$

$158 \quad 12.6$

$504 \quad 40.2$

$117 \quad 56.8$

$119 \quad 9.5$

$\begin{array}{rr}8 & 3.9 \\ 61 & 11 .\end{array}$

$17 \quad 8.3$

$267 \quad 48.5$

30938.8

$173 \quad 63.1$

10513

$14 \quad 2.5$

$\begin{array}{llll}5 & 13.2 & 55 & 6.9\end{array}$

$\begin{array}{rrrr}21 & 7.7 & 8 & 2.9\end{array}$

$\begin{array}{llll}64 & 10.4 & 39 & 6.3\end{array}$

$1655 \quad 44.8$

$417 \quad 11.3$

$252 \quad 6.8$

Greater Southern

Griffith $B$

$\begin{array}{llll}42 & 9.3 & 13 & 2.9\end{array}$

$\begin{array}{llll}81 & 11.6 & 55 & 7.9\end{array}$

$\begin{array}{llll}34 & 6.0 & 33 & 5.8\end{array}$

$\begin{array}{lllr}34 & 11.1 & 42 & 13.7\end{array}$

$\begin{array}{llll}21 & 8.2 & 10 & 3.9\end{array}$

$\begin{array}{llll}192 & 11.9 & 81 & 5.0\end{array}$

$\begin{array}{llll}404 & 10.4 & 234 & 6.0\end{array}$

$\begin{array}{lrrr}2 & 1.8 & 0 & 0.0\end{array}$

$\begin{array}{llll}113 & 15.8 & 47 & 6.6\end{array}$

$\begin{array}{llll}12 & 1.7 & 66 & 9.2\end{array}$

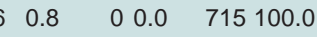

$\begin{array}{llllllll}95 & 10.1 & 126 & 6.5 & 22 & 1.1 & 310 & 16.0\end{array}$

$\begin{array}{llllllll}18 & 8.4 & 17 & 7.9 & 2 & 0.9 & 22 & 10.2\end{array}$

$\begin{array}{llllllll}181 & 18.1 & 83 & 8.3 & 13 & 1.3 & 292 & 29.2\end{array}$

$\begin{array}{llll}0 & 0.0 & 1936100.0\end{array}$

$\begin{array}{llllll}0 & 0.0 & 0 & 0.0 & 215 & 100.0\end{array}$

$\begin{array}{llllllll}597 & 16.3 & 251 & 6.8 & 63 & 1.7 & 489 & 13.3\end{array}$

$\begin{array}{llllllll}202 & 9.1 & 197 & 8.8 & 28 & 1.3 & 200 & 9.0\end{array}$

$\begin{array}{llllllll}109 & 14.0 & 41 & 5.3 & 9 & 1.2 & 124 & 16.0\end{array}$

$\begin{array}{llllllll}272 & 23.5 & 80 & 6.9 & 9 & 0.8 & 256 & 22.1\end{array}$

$\begin{array}{llllllll}222 & 32.4 & 87 & 12.7 & 9 & 1.3 & 142 & 20.7\end{array}$

$\begin{array}{llllllll}336 & 21.3 & 188 & 11.9 & 30 & 1.9 & 189 & 12.0\end{array}$

$\begin{array}{llllllll}451 & 26.5 & 119 & 7.0 & 43 & 2.5 & 208 & 12.2\end{array}$

$\begin{array}{llllllll}26 & 17.9 & 23 & 15.9 & 0 & 0.0 & 15 & 10.3\end{array}$

$\begin{array}{llllll}0 & 0.0 & 0 & 0.0 & 1001 & 100.0\end{array}$

$\begin{array}{llllllll}722 & 17.2 & 1259 & 8.0 & 240 & 1.5 & 2313 & 14.6\end{array}$

$20 \quad 0.5$

$00.0 \quad 3673100.0$

$\begin{array}{llllll}14 & 0.6 & 0 & 0.0 & 2229 & 100.0\end{array}$

$\begin{array}{llllll}5 & 0.6 & 0 & 0.0 & 776 & 100.0\end{array}$

$\begin{array}{llllll}0 & 0.0 & 0 & 0.0 & 1156 & 100.0\end{array}$

$\begin{array}{llllll}0 & 0.0 & 0 & 0.0 & 685100.0\end{array}$

$\begin{array}{llllll}1 & 0.1 & 0 & 0.0 & 1579 & 100.0\end{array}$

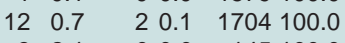

$\begin{array}{llllll}3 & 2.1 & 0 & 0.0 & 145100.0\end{array}$

20.015814100 .0

$\begin{array}{llllllll}68 & 16.9 & 57 & 14.1 & 4 & 1.0 & 41 & 10.2\end{array}$

$\begin{array}{lllll}0 & 0.0 & 0 & 0.0 & 403 \\ 100.0\end{array}$

$\begin{array}{llllllll}58 & 13.3 & 104 & 8.8 & 28 & 2.4 & 135 & 11.4\end{array}$

$\begin{array}{llllllll}42 & 11.3 & 32 & 8.6 & 7 & 1.9 & 47 & 12.7\end{array}$

$\begin{array}{llllllll}138 & 16.3 & 61 & 7.2 & 25 & 2.9 & 114 & 13.4\end{array}$

$\begin{array}{llllllll}117 & 16.0 & 60 & 8.2 & 12 & 1.6 & 127 & 17.3\end{array}$

$\begin{array}{llllllll}26 & 9.4 & 34 & 12.3 & 2 & 0.7 & 28 & 10.1\end{array}$

$\begin{array}{llllllll}117 & 16.4 & 79 & 11.1 & 12 & 1.7 & 66 & 9.2\end{array}$

$\begin{array}{llllllll}48 & 9.8 & 14 & 2.9 & 17 & 3.5 & 27 & 5.5\end{array}$

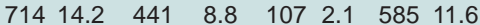

$\begin{array}{llllll}2 & 0.2 & 0 & 0.0 & 1188 & 100.0\end{array}$

$\begin{array}{llllll}0 & 0 & 0 & 0 & 0.0 & 371 \\ 100 & 0\end{array}$

$\begin{array}{llllll}1 & 0.1 & 0 & 0.0 & 848 & 100.0\end{array}$

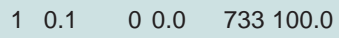

$\begin{array}{llllll}0 & 0.0 & 0 & 0.0 & 277 & 100.0\end{array}$

$\begin{array}{llllll}0 & 0.0 & 0 & 0.0 & 714 & 100.0\end{array}$

$\begin{array}{llllll}0 & 0.0 & 0 & 0.0 & 491 & 100.0\end{array}$

$\begin{array}{llllllll}122 & 9.7 & 93 & 7.4 & 36 & 2.9 & 220 & 17.6\end{array}$

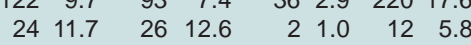

$4 \quad 0.1$

0.05025100 .0

$\begin{array}{llllll}0 & 0.0 & 0 & 0.0 & 206 & 100.0\end{array}$

$\begin{array}{lllllllllllll}11.7 & 68 & 12.4 & 4 & 0.7 & 26 & 4.7 & 0 & 0.0 & 0 & 0.0 & 550 & 100.0\end{array}$

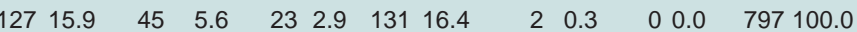

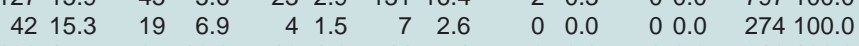

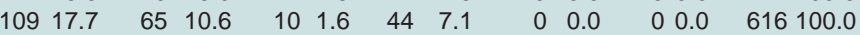

$\begin{array}{llllllllllllll}534 & 14.4 & 316 & 8.5 & 79 & 2.1 & 440 & 11.9 & 3 & 0.1 & 0 & 0.0 & 3696 & 100.0\end{array}$

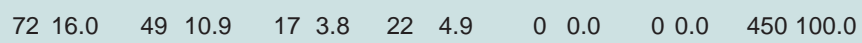

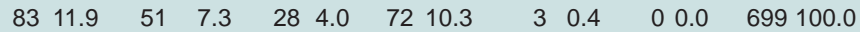

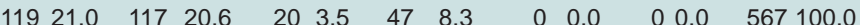

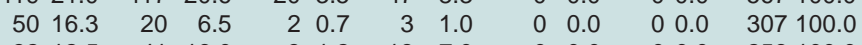

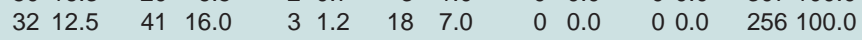

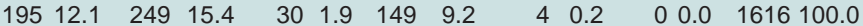

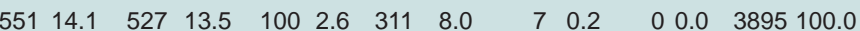

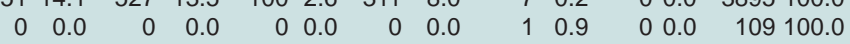

$\begin{array}{ll}1761 & 45.2 \\ 106 & 97.2\end{array}$

2890.3

20.085032100 .0

TOTAL NSW

$\begin{array}{llllllllllll}38110 & 44.8 & 5992 & 7.0 & 7258 & 8.5 & 12820 & 15.1 & 7265 & 8.5 & 1331 & 1.611965\end{array}$

Source: NSW Midwives Data Collection (HOIST). Centre for Epidemiology and Research, NSW Department of Health.

\# Hospitals with more than 200 deliveries are identified individually. All hospitals include all public and private hospitals.

\#\# This category includes other forms of induction such as Foley's catheter.

* Royal Prince Alfred and Canterbury Hospitals supply data electronically and report augmentation by oxytocin-prostaglandin only. 


\section{Type of delivery in selected hospitals}

Table 116 gives type of delivery for individual hospitals where the number of reported confinements exceeded 200 in 2003, totals for all hospitals within each health area and the NSW total.

\section{TABLE 116}

CONFINEMENTS BY TYPE OF DELIVERY AND HOSPITAL, NSW 2003"

\begin{tabular}{|c|c|c|c|c|c|c|c|c|c|c|c|c|c|c|c|c|}
\hline \multirow{3}{*}{$\begin{array}{l}\text { Health Area and } \\
\text { Hospital }\end{array}$} & \multirow{2}{*}{\multicolumn{2}{|c|}{$\begin{array}{l}\text { Normal } \\
\text { vaginal }\end{array}$}} & \multirow{2}{*}{\multicolumn{2}{|c|}{ Forceps }} & \multirow{2}{*}{\multicolumn{2}{|c|}{$\begin{array}{l}\text { Vacuum } \\
\text { extraction }\end{array}$}} & \multicolumn{4}{|c|}{ Type of delivery } & \multirow{2}{*}{\multicolumn{2}{|c|}{$\begin{array}{l}\text { Emergency } \\
\text { caesarean }\end{array}$}} & \multirow{2}{*}{\multicolumn{2}{|c|}{ Not stated }} & \multirow{2}{*}{\multicolumn{2}{|c|}{ TOTAL }} \\
\hline & & & & & & & & & & $\begin{array}{l}\text { tive } \\
\text { arean }\end{array}$ & & & & & & \\
\hline & No. & $\%$ & No. & $\%$ & No. & $\%$ & No. & $\%$ & No. & $\%$ & No. & $\%$ & No. & $\%$ & No. & $\%$ \\
\hline \multicolumn{17}{|l|}{ Sydney South West } \\
\hline Canterbury & 996 & 71.4 & 22 & 1.6 & 90 & 6.5 & 5 & 0.4 & 163 & 11.7 & 118 & 8.5 & 0 & 0.0 & 1394 & 100.0 \\
\hline Royal Prince Alfred & 2547 & 63.0 & 82 & 2.0 & 319 & 7.9 & 14 & 0.3 & 580 & 14.3 & 500 & 12.4 & 0 & 0.0 & 4042 & 100.0 \\
\hline Camden & 453 & 85.0 & 27 & 5.1 & 2 & 0.4 & 1 & 0.2 & 7 & 1.3 & 43 & 8.1 & 0 & 0.0 & 533 & 100.0 \\
\hline Fairfield & 1378 & 76.2 & 24 & 1.3 & 115 & 6.4 & 10 & 0.6 & 167 & 9.2 & 115 & 6.4 & 0 & 0.0 & 1809 & 100.0 \\
\hline Liverpool & 2220 & 71.6 & 21 & 0.7 & 190 & 6.1 & 24 & 0.8 & 349 & 11.3 & 296 & 9.5 & 0 & 0.0 & 3100 & 100.0 \\
\hline Campbelltown & 1404 & 69.5 & 5 & 0.2 & 133 & 6.6 & 5 & 0.2 & 265 & 13.1 & 209 & 10.3 & 0 & 0.0 & 2021 & 100.0 \\
\hline \multicolumn{17}{|c|}{ Sydney Southwest } \\
\hline Private & 627 & 54.0 & 32 & 2.8 & 176 & 15.1 & 4 & 0.3 & 206 & 17.7 & 117 & 10.1 & 0 & 0.0 & 1162 & 100.0 \\
\hline Bowral & 402 & 61.5 & 31 & 4.7 & 92 & 14.1 & 2 & 0.3 & 75 & 11.5 & 52 & 8.0 & 0 & 0.0 & 654 & 100.0 \\
\hline Other Area hospitals & 0 & 0.0 & 0 & 0.0 & 0 & 0.0 & 1 & 50.0 & 0 & 0.0 & 1 & 50.0 & 0 & 0.0 & 2 & 100.0 \\
\hline ALL HOSPITALS & 11366 & 68.8 & 279 & 1.7 & 1223 & 7.4 & 82 & 0.5 & 2005 & 12.1 & 1577 & 9.5 & 0 & 0.0 & 16532 & 100.0 \\
\hline \multicolumn{17}{|l|}{ Northern Sydney \& } \\
\hline Hornsby & 593 & 65.2 & 37 & 4.1 & 64 & 7.0 & 2 & 0.2 & 129 & 14.2 & 84 & 9.2 & 0 & 0.0 & 909 & 100.0 \\
\hline Manly & 506 & 64.8 & 16 & 2.0 & 48 & 6.1 & 2 & 0.3 & 105 & 13.4 & 104 & 13.3 & 0 & 0.0 & 781 & 100.0 \\
\hline Mona Vale & 363 & 62.3 & 7 & 1.2 & 73 & 12.5 & 5 & 0.9 & 71 & 12.2 & 64 & 11.0 & 0 & 0.0 & 583 & 100.0 \\
\hline Royal North Shore & 895 & 54.9 & 77 & 4.7 & 94 & 5.8 & 13 & 0.8 & 294 & 18.0 & 256 & 15.7 & 0 & 0.0 & 1629 & 100.0 \\
\hline Ryde & 314 & 70.9 & 22 & 5.0 & 8 & 1.8 & 2 & 0.5 & 66 & 14.9 & 31 & 7.0 & 0 & 0.0 & 443 & 100.0 \\
\hline Mater, North Sydney & 856 & 41.4 & 78 & 3.8 & 272 & 13.2 & 2 & 0.1 & 568 & 27.5 & 290 & 14.0 & 0 & 0.0 & 2066 & 100.0 \\
\hline North Shore Private & 990 & 44.3 & 82 & 3.7 & 237 & 10.6 & 5 & 0.2 & 593 & 26.5 & 330 & 14.8 & 0 & 0.0 & 2237 & 100.0 \\
\hline Sydney Adventist & 1260 & 54.8 & 145 & 6.3 & 158 & 6.9 & 8 & 0.3 & 474 & 20.6 & 253 & 11.0 & 0 & 0.0 & 2298 & 100.0 \\
\hline Gosford & 1336 & 62.8 & 17 & 0.8 & 202 & 9.5 & 8 & 0.4 & 259 & 12.2 & 306 & 14.4 & 0 & 0.0 & 2128 & 100.0 \\
\hline Wyong & 327 & 92.1 & 1 & 0.3 & 14 & 3.9 & 0 & 0.0 & 5 & 1.4 & 8 & 2.3 & 0 & 0.0 & 355 & 100.0 \\
\hline North Gosford Private & e 417 & 47.4 & 21 & 2.4 & 110 & 12.5 & 0 & 0.0 & 246 & 28.0 & 86 & 9.8 & 0 & 0.0 & 880 & 100.0 \\
\hline ALL HOSPITALS & 7857 & 54.9 & 503 & 3.5 & 1280 & 8.9 & 47 & 0.3 & 2810 & 19.6 & 1812 & 12.7 & 0 & 0.0 & 14309 & 100.0 \\
\hline \multicolumn{17}{|l|}{ Sydney West } \\
\hline Auburn & 934 & 78.8 & 20 & 1.7 & 35 & 3.0 & 4 & 0.3 & 104 & 8.8 & 89 & 7.5 & 0 & 0.0 & 1186 & 100.0 \\
\hline Blacktown & 1689 & 68.5 & 119 & 4.8 & 98 & 4.0 & 6 & 0.2 & 256 & 10.4 & 296 & 12.0 & 0 & 0.0 & 2464 & 100.0 \\
\hline Westmead & 2477 & 63.5 & 255 & 6.5 & 113 & 2.9 & 49 & 1.3 & 504 & 12.9 & 504 & 12.9 & 0 & 0.0 & 3902 & 100.0 \\
\hline The Hills Private & 804 & 61.0 & 105 & 8.0 & 65 & 4.9 & 5 & 0.4 & 204 & 15.5 & 134 & 10.2 & 0 & 0.0 & 1317 & 100.0 \\
\hline Westmead Private & 875 & 56.0 & 127 & 8.1 & 112 & 7.2 & 5 & 0.3 & 247 & 15.8 & 197 & 12.6 & 0 & 0.0 & 1563 & 100.0 \\
\hline Blue Mountains & 216 & 68.6 & 3 & 1.0 & 26 & 8.3 & 2 & 0.6 & 41 & 13.0 & 27 & 8.6 & 0 & 0.0 & 315 & 100.0 \\
\hline Nepean & 2056 & 63.5 & 78 & 2.4 & 253 & 7.8 & 18 & 0.6 & 442 & 13.6 & 393 & 12.1 & 0 & 0.0 & 3240 & 100.0 \\
\hline Hawkesbury & 582 & 66.7 & 48 & 5.5 & 25 & 2.9 & 0 & 0.0 & 106 & 12.1 & 112 & 12.8 & 0 & 0.0 & 873 & 100.0 \\
\hline Nepean Private & 433 & 49.8 & 41 & 4.7 & 56 & 6.4 & 2 & 0.2 & 184 & 21.2 & 153 & 17.6 & 0 & 0.0 & 869 & 100.0 \\
\hline Other Area hospitals & 131 & 62.4 & 0 & 0.0 & 16 & 7.6 & 0 & 0.0 & 29 & 13.8 & 34 & 16.2 & 0 & 0.0 & 210 & 100.0 \\
\hline ALL HOSPITALS & 10197 & 64.0 & 796 & 5.0 & 799 & 5.0 & 91 & 0.6 & 2117 & 13.3 & 1939 & 12.2 & 0 & 0.0 & 15939 & 100.0 \\
\hline \multicolumn{17}{|c|}{ Hunter \& New England } \\
\hline Maitland & 983 & 66.0 & 4 & 0.3 & 107 & 7.2 & 4 & 0.3 & 230 & 15.4 & 162 & 10.9 & 0 & 0.0 & 1490 & 100.0 \\
\hline Muswellbrook & 161 & 71.6 & 0 & 0.0 & 19 & 8.4 & 1 & 0.4 & 23 & 10.2 & 21 & 9.3 & 0 & 0.0 & 225 & 100.0 \\
\hline Belmont & 432 & 70.1 & 10 & 1.6 & 34 & 5.5 & 0 & 0.0 & 77 & 12.5 & 63 & 10.2 & 0 & 0.0 & 616 & 100.0 \\
\hline John Hunter & 2097 & 67.3 & 66 & 2.1 & 218 & 7.0 & 33 & 1.1 & 390 & 12.5 & 314 & 10.1 & 0 & 0.0 & 3118 & 100.0 \\
\hline Christo Road Private & 619 & 53.1 & 61 & 5.2 & 100 & 8.6 & 0 & 0.0 & 224 & 19.2 & 161 & 13.8 & 0 & 0.0 & 1165 & 100.0 \\
\hline Manning Base & 470 & 74.0 & 7 & 1.1 & 38 & 6.0 & 5 & 0.8 & 53 & 8.3 & 62 & 9.8 & 0 & 0.0 & 635 & 100.0 \\
\hline Armidale & 338 & 77.0 & 3 & 0.7 & 20 & 4.6 & 3 & 0.7 & 48 & 10.9 & 27 & 6.2 & 0 & 0.0 & 439 & 100.0 \\
\hline Inverell & 141 & 67.1 & 10 & 4.8 & 0 & 0.0 & 0 & 0.0 & 40 & 19.0 & 19 & 9.0 & 0 & 0.0 & 210 & 100.0 \\
\hline Tamworth Base & 404 & 65.8 & 8 & 1.3 & 41 & 6.7 & 5 & 0.8 & 97 & 15.8 & 59 & 9.6 & 0 & 0.0 & 614 & 100.0 \\
\hline Other Area hospitals & 845 & 70.4 & 20 & 1.7 & 67 & 5.6 & 7 & 0.6 & 185 & 15.4 & 77 & 6.4 & 0 & 0.0 & 1201 & 100.0 \\
\hline ALL HOSPITALS & 6490 & 66.8 & 189 & 1.9 & 644 & 6.6 & 58 & 0.6 & 1367 & 14.1 & 965 & 9.9 & 0 & 0.0 & 9713 & 100.0 \\
\hline
\end{tabular}


TABLE 116 (continued)

CONFINEMENTS BY TYPE OF DELIVERY AND HOSPITAL, NSW 2003\#

\begin{tabular}{|c|c|c|c|c|c|c|c|c|c|c|c|c|c|c|c|c|}
\hline \multirow[t]{2}{*}{$\begin{array}{l}\text { Health Area and } \\
\text { Hospital }\end{array}$} & \multicolumn{2}{|c|}{ Normal } & \multicolumn{2}{|c|}{$\begin{array}{l}\text { Forceps } \\
\text { vaginal }\end{array}$} & \multicolumn{2}{|c|}{$\begin{array}{l}\text { Vacuum } \\
\text { extraction }\end{array}$} & \multicolumn{2}{|c|}{$\begin{array}{c}\text { Type of delivery } \\
\text { Vaginal } \\
\text { breech }\end{array}$} & \multicolumn{2}{|c|}{$\begin{array}{c}\text { Elective } \\
\text { caesarean }\end{array}$} & \multicolumn{2}{|c|}{$\begin{array}{l}\text { Emergency } \\
\text { caesarean }\end{array}$} & \multicolumn{2}{|c|}{ Not stated } & \multicolumn{2}{|c|}{ TOTAL } \\
\hline & No. & $\%$ & No. & $\%$ & No. & $\%$ & No. & $\%$ & No. & $\%$ & No. & $\%$ & No. & $\%$ & No. & $\%$ \\
\hline \multicolumn{17}{|l|}{$\begin{array}{l}\text { South Eastern Sydney \& } \\
\text { Illawarra }\end{array}$} \\
\hline Shoalhaven & 464 & 64.9 & 29 & 4.1 & 11 & 1.5 & 1 & 0.1 & 113 & 15.8 & 97 & 13.6 & 0 & 0.0 & 715 & 100.0 \\
\hline Wollongong & 1300 & 67.1 & 31 & 1.6 & 177 & 9.1 & 2 & 0.1 & 195 & 10.1 & 231 & 11.9 & 0 & 0.0 & 1936 & 100.0 \\
\hline Shellharbour & 166 & 77.2 & 2 & 0.9 & 8 & 3.7 & 1 & 0.5 & 18 & 8.4 & 20 & 9.3 & 0 & 0.0 & 215 & 100.0 \\
\hline Illawarra Private & 523 & 52.2 & 11 & 1.1 & 167 & 16.7 & 0 & 0.0 & 181 & 18.1 & 119 & 11.9 & 0 & 0.0 & 1001 & 100.0 \\
\hline \multicolumn{17}{|l|}{ Royal Hospital } \\
\hline St. George & 1453 & 65.2 & 65 & 2.9 & 185 & 8.3 & 8 & 0.4 & 202 & 9.1 & 316 & 14.2 & 0 & 0.0 & 2229 & 100.0 \\
\hline Sutherland & 538 & 69.3 & 21 & 2.7 & 39 & 5.0 & 2 & 0.3 & 109 & 14.0 & 67 & 8.6 & 0 & 0.0 & 776 & 100.0 \\
\hline Hurstville Community & 506 & 43.8 & 51 & 4.4 & 165 & 14.3 & 1 & 0.1 & 272 & 23.5 & 161 & 13.9 & 0 & 0.0 & 1156 & 100.0 \\
\hline Kareena Private & 264 & 38.5 & 80 & 11.7 & 37 & 5.4 & 0 & 0.0 & 222 & 32.4 & 82 & 12.0 & 0 & 0.0 & 685 & 100.0 \\
\hline \multicolumn{17}{|l|}{ Prince of Wales } \\
\hline Private & 787 & 46.2 & 62 & 3.6 & 176 & 10.3 & 3 & 0.2 & 451 & 26.5 & 215 & 12.6 & 10 & 0.6 & 1704 & 100.0 \\
\hline Other Area hospitals & 100 & 69.0 & 4 & 2.8 & 5 & 3.4 & 0 & 0.0 & 26 & 17.9 & 10 & 6.9 & 0 & 0.0 & 145 & 100.0 \\
\hline ALL HOSPITALS & 9045 & 57.2 & 661 & 4.2 & 1280 & 8.1 & 42 & 0.3 & 2722 & 17.2 & 2054 & 13.0 & 10 & 0.1 & 15814 & 100.0 \\
\hline \multicolumn{17}{|l|}{ North Coast } \\
\hline Grafton Base & 238 & 59.1 & 13 & 3.2 & 14 & 3.5 & 1 & 0.2 & 68 & 16.9 & 69 & 17.1 & 0 & 0.0 & 403 & 100.0 \\
\hline Lismore Base & 784 & 66.0 & 29 & 2.4 & 28 & 2.4 & 7 & 0.6 & 158 & 13.3 & 182 & 15.3 & 0 & 0.0 & 1188 & 100.0 \\
\hline Murwillumbah & 236 & 63.6 & 5 & 1.3 & 30 & 8.1 & 1 & 0.3 & 42 & 11.3 & 57 & 15.4 & 0 & 0.0 & 371 & 100.0 \\
\hline Tweed Heads & 571 & 67.3 & 6 & 0.7 & 49 & 5.8 & 4 & 0.5 & 138 & 16.3 & 80 & 9.4 & 0 & 0.0 & 848 & 100.0 \\
\hline Coffs Harbour Base & 494 & 67.4 & 23 & 3.1 & 24 & 3.3 & 3 & 0.4 & 117 & 16.0 & 72 & 9.8 & 0 & 0.0 & 733 & 100.0 \\
\hline Kempsey & 215 & 77.6 & 3 & 1.1 & 5 & 1.8 & 0 & 0.0 & 26 & 9.4 & 28 & 10.1 & 0 & 0.0 & 277 & 100.0 \\
\hline Port Macquarie Base & 429 & 60.1 & 39 & 5.5 & 24 & 3.4 & 3 & 0.4 & 117 & 16.4 & 102 & 14.3 & 0 & 0.0 & 714 & 100.0 \\
\hline Other Area hospitals & 388 & 79.0 & 19 & 3.9 & 19 & 3.9 & 3 & 0.6 & 48 & 9.8 & 14 & 2.9 & 0 & 0.0 & 491 & 100.0 \\
\hline ALL HOSPITALS & 3355 & 66.8 & 137 & 2.7 & 193 & 3.8 & 22 & 0.4 & 714 & 14.2 & 604 & 12.0 & 0 & 0.0 & 5025 & 100.0 \\
\hline \multicolumn{17}{|l|}{ Greater Western } \\
\hline Dubbo Base & 909 & 72.5 & 45 & 3.6 & 46 & 3.7 & 7 & 0.6 & 122 & 9.7 & 124 & 9.9 & 0 & 0.0 & 1253 & 100.0 \\
\hline Mudgee & 150 & 72.8 & 0 & 0.0 & 7 & 3.4 & 1 & 0.5 & 24 & 11.7 & 24 & 11.7 & 0 & 0.0 & 206 & 100.0 \\
\hline Bathurst Base & 328 & 59.6 & 10 & 1.8 & 22 & 4.0 & 2 & 0.4 & 110 & 20.0 & 78 & 14.2 & 0 & 0.0 & 550 & 100.0 \\
\hline Orange Base & 519 & 65.1 & 35 & 4.4 & 40 & 5.0 & 2 & 0.3 & 127 & 15.9 & 74 & 9.3 & 0 & 0.0 & 797 & 100.0 \\
\hline Broken Hill Base & 200 & 73.0 & 2 & 0.7 & 2 & 0.7 & 1 & 0.4 & 42 & 15.3 & 27 & 9.9 & 0 & 0.0 & 274 & 100.0 \\
\hline Other Area hospitals & 418 & 67.9 & 9 & 1.5 & 19 & 3.1 & 1 & 0.2 & 109 & 17.7 & 60 & 9.7 & 0 & 0.0 & 616 & 100.0 \\
\hline ALL HOSPITALS & 2524 & 68.3 & 101 & 2.7 & 136 & 3.7 & 14 & 0.4 & 534 & 14.4 & 387 & 10.5 & 0 & 0.0 & 3696 & 100.0 \\
\hline \multicolumn{17}{|l|}{ Greater Southern } \\
\hline Griffith Base & 279 & 62.0 & 24 & 5.3 & 19 & 4.2 & 1 & 0.2 & 72 & 16.0 & 55 & 12.2 & 0 & 0.0 & 450 & 100.0 \\
\hline \multicolumn{17}{|l|}{ Calvary, Wagga } \\
\hline Wagga & 281 & 49.6 & 46 & 8.1 & 50 & 8.8 & 0 & 0.0 & 119 & 21.0 & 71 & 12.5 & 0 & 0.0 & 567 & 100.0 \\
\hline Goulburn Base & 164 & 53.4 & 35 & 11.4 & 18 & 5.9 & 3 & 1.0 & 50 & 16.3 & 37 & 12.1 & 0 & 0.0 & 307 & 100.0 \\
\hline Queanbeyan & 189 & 73.8 & 9 & 3.5 & 13 & 5.1 & 1 & 0.4 & 32 & 12.5 & 12 & 4.7 & 0 & 0.0 & 256 & 100.0 \\
\hline Other Area hospitals & 1103 & 68.3 & 58 & 3.6 & 99 & 6.1 & 6 & 0.4 & 195 & 12.1 & 155 & 9.6 & 0 & 0.0 & 1616 & 100.0 \\
\hline ALL HOSPITALS & 2483 & 63.7 & 209 & 5.4 & 233 & 6.0 & 13 & 0.3 & 551 & 14.1 & 406 & 10.4 & 0 & 0.0 & 3895 & 100.0 \\
\hline Other/Not stated & 107 & 98.2 & 0 & 0.0 & 0 & 0.0 & 2 & 1.8 & 0 & 0.0 & 0 & 0.0 & 0 & 0.0 & 109 & 100.0 \\
\hline TOTAL NSW & 53424 & 62.8 & 2875 & 3.4 & 5788 & 6.8 & 371 & 0.41 & 12820 & 15.1 & 9744 & 11.5 & 10 & 0.0 & 85032 & 100.0 \\
\hline
\end{tabular}

Source: NSW Midwives Data Collection (HOIST). Centre for Epidemiology and Research, NSW Department of Health.

\# Hospitals with more than 200 deliveries are identified individually. All hospitals include all public and private hospitals. 


\section{Pain relief in selected hospitals}

Table 117 gives type of pain relief provided to women for individual hospitals where the number of reported confinements exceeded 200 in 2003, totals for all hospitals within each health area and the NSW total. In addition to the types of pain relief listed a further 23,695 (27.9 per cent) women were reported to have received local anaesthetic to the perineum, and 778 ( 0.9 per cent $)$ received a pudendal block.

\section{TABLE 117}

CONFINEMENTS BY TYPE OF PAIN RELIEF AND HOSPITAL, NSW 2003"

\begin{tabular}{|c|c|c|c|c|c|c|c|c|c|c|c|c|c|c|}
\hline \multirow{3}{*}{$\begin{array}{l}\text { Health Area and } \\
\text { Hospital }\end{array}$} & \multirow{2}{*}{\multicolumn{2}{|c|}{ Epidural }} & \multirow{2}{*}{\multicolumn{2}{|c|}{$\begin{array}{c}\text { General } \\
\text { anaesthetic }\end{array}$}} & \multicolumn{4}{|c|}{ Type of pain relief } & \multirow{2}{*}{\multicolumn{2}{|c|}{ Spinal }} & \multirow{2}{*}{\multicolumn{2}{|c|}{ Nil }} & \multirow{2}{*}{\multicolumn{2}{|c|}{ TOTAL }} \\
\hline & & & & & & $\begin{array}{l}\text { M } \\
\text { otics }\end{array}$ & & & & & & & & \\
\hline & No. & $\%$ & No. & $\%$ & No. & $\%$ & No. & $\%$ & No. & $\%$ & No. & $\%$ & No. & $\%$ \\
\hline \multicolumn{15}{|l|}{ Sydney South West } \\
\hline Canterbury & 215 & 15.4 & 100 & 7.2 & 465 & 33.4 & 575 & 41.2 & 118 & 8.5 & 229 & 16.4 & 1394 & 100.0 \\
\hline Royal Prince Alfred & 1341 & 33.2 & 227 & 5.6 & 1704 & 42.2 & 1356 & 33.5 & 350 & 8.7 & 517 & 12.8 & 4042 & 100.0 \\
\hline Camden & 3 & 0.6 & 20 & 3.8 & 185 & 34.7 & 345 & 64.7 & 34 & 6.4 & 83 & 15.6 & 533 & 100.0 \\
\hline Fairfield & 78 & 4.3 & 212 & 11.7 & 542 & 30.0 & 945 & 52.2 & 56 & 3.1 & 281 & 15.5 & 1809 & 100.0 \\
\hline Liverpool & 475 & 15.3 & 296 & 9.5 & 1346 & 43.4 & 1581 & 51.0 & 245 & 7.9 & 278 & 9.0 & 3100 & 100.0 \\
\hline Campbelltown & 212 & 10.5 & 164 & 8.1 & 819 & 40.5 & 1217 & 60.2 & 271 & 13.4 & 179 & 8.9 & 2021 & 100.0 \\
\hline Bankstown-Lidcombe & 133 & 7.3 & 130 & 7.2 & 380 & 20.9 & 1077 & 59.3 & 170 & 9.4 & 203 & 11.2 & 1815 & 100.0 \\
\hline Sydney Southwest Private & 385 & 33.1 & 70 & 6.0 & 411 & 35.4 & 707 & 60.8 & 67 & 5.8 & 35 & 3.0 & 1162 & 100.0 \\
\hline Bowral & 154 & 23.5 & 20 & 3.1 & 274 & 41.9 & 320 & 48.9 & 71 & 10.9 & 55 & 8.4 & 654 & 100.0 \\
\hline Other Area hospitals & 1 & 50.0 & 0 & 0.0 & 1 & 50.0 & 1 & 50.0 & 0 & 0.0 & 0 & 0.0 & 2 & 100.0 \\
\hline ALL HOSPITALS & 2997 & 18.1 & 1239 & 7.5 & 6127 & 37.1 & 8124 & 49.1 & 1382 & 8.4 & 1860 & 11.3 & 16532 & 100.0 \\
\hline \multicolumn{15}{|l|}{$\begin{array}{l}\text { Northern Sydney \& } \\
\text { Central Coast }\end{array}$} \\
\hline Hornsby & 349 & 38.4 & 48 & 5.3 & 197 & 21.7 & 476 & 52.4 & 52 & 5.7 & 67 & 7.4 & 909 & 100.0 \\
\hline Manly & 173 & 22.2 & 17 & 2.2 & 207 & 26.5 & 374 & 47.9 & 159 & 20.4 & 75 & 9.6 & 781 & 100.0 \\
\hline Mona Vale & 181 & 31.0 & 18 & 3.1 & 293 & 50.3 & 252 & 43.2 & 83 & 14.2 & 47 & 8.1 & 583 & 100.0 \\
\hline Royal North Shore & 486 & 29.8 & 74 & 4.5 & 365 & 22.4 & 808 & 49.6 & 391 & 24.0 & 73 & 4.5 & 1629 & 100.0 \\
\hline Ryde & 69 & 15.6 & 23 & 5.2 & 119 & 26.9 & 217 & 49.0 & 70 & 15.8 & 50 & 11.3 & 443 & 100.0 \\
\hline Mater, North Sydney & 1250 & 60.5 & 34 & 1.6 & 230 & 11.1 & 694 & 33.6 & 191 & 9.2 & 48 & 2.3 & 2066 & 100.0 \\
\hline North Shore Private & 1230 & 55.0 & 61 & 2.7 & 185 & 8.3 & 684 & 30.6 & 521 & 23.3 & 61 & 2.7 & 2237 & 100.0 \\
\hline Sydney Adventist & 1327 & 57.7 & 76 & 3.3 & 285 & 12.4 & 822 & 35.8 & 153 & 6.7 & 76 & 3.3 & 2298 & 100.0 \\
\hline Gosford & 478 & 22.5 & 123 & 5.8 & 729 & 34.3 & 969 & 45.5 & 362 & 17.0 & 90 & 4.2 & 2128 & 100.0 \\
\hline Wyong & 0 & 0.0 & 14 & 3.9 & 93 & 26.2 & 142 & 40.0 & 2 & 0.6 & 35 & 9.9 & 355 & 100.0 \\
\hline North Gosford Private & 233 & 26.5 & 22 & 2.5 & 169 & 19.2 & 355 & 40.3 & 243 & 27.6 & 56 & 6.4 & 880 & 100.0 \\
\hline ALL HOSPITALS & 5776 & 40.4 & 510 & 3.6 & 2872 & 20.1 & 5793 & 40.5 & 2227 & 15.6 & 678 & 4.7 & 14309 & 100.0 \\
\hline \multicolumn{15}{|l|}{ Sydney West } \\
\hline Auburn & 79 & 6.7 & 104 & 8.8 & 288 & 24.3 & 546 & 46.0 & 70 & 5.9 & 253 & 21.3 & 1186 & 100.0 \\
\hline Blacktown & 536 & 21.8 & 173 & 7.0 & 487 & 19.8 & 1225 & 49.7 & 255 & 10.3 & 319 & 12.9 & 2464 & 100.0 \\
\hline Westmead & 1325 & 34.0 & 262 & 6.7 & 715 & 18.3 & 1766 & 45.3 & 465 & 11.9 & 347 & 8.9 & 3902 & 100.0 \\
\hline The Hills Private & 685 & 52.0 & 42 & 3.2 & 215 & 16.3 & 506 & 38.4 & 48 & 3.6 & 62 & 4.7 & 1317 & 100.0 \\
\hline Westmead Private & 601 & 38.5 & 62 & 4.0 & 308 & 19.7 & 802 & 51.3 & 224 & 14.3 & 116 & 7.4 & 1563 & 100.0 \\
\hline Blue Mountains & 60 & 19.0 & 11 & 3.5 & 66 & 21.0 & 114 & 36.2 & 46 & 14.6 & 54 & 17.1 & 315 & 100.0 \\
\hline Nepean & 796 & 24.6 & 298 & 9.2 & 1052 & 32.5 & 1796 & 55.4 & 405 & 12.5 & 274 & 8.5 & 3240 & 100.0 \\
\hline Hawkesbury & 62 & 7.1 & 41 & 4.7 & 211 & 24.2 & 489 & 56.0 & 171 & 19.6 & 112 & 12.8 & 873 & 100.0 \\
\hline Nepean Private & 241 & 27.7 & 43 & 4.9 & 220 & 25.3 & 454 & 52.2 & 203 & 23.4 & 72 & 8.3 & 869 & 100.0 \\
\hline Other Area hospitals & 61 & 29.0 & 5 & 2.4 & 39 & 18.6 & 95 & 45.2 & 20 & 9.5 & 36 & 17.1 & 210 & 100.0 \\
\hline ALL HOSPITALS & 4446 & 27.9 & 1041 & 6.5 & 3601 & 22.6 & 7793 & 48.9 & 1907 & 12.0 & 1645 & 10.3 & 15939 & 100.0 \\
\hline \multicolumn{15}{|l|}{ Hunter \& New England } \\
\hline Maitland & 122 & 8.2 & 80 & 5.4 & 473 & 31.7 & 814 & 54.6 & 297 & 19.9 & 184 & 12.3 & 1490 & 100.0 \\
\hline Muswellbrook & 3 & 1.3 & 0 & 0.0 & 60 & 26.7 & 103 & 45.8 & 43 & 19.1 & 60 & 26.7 & 225 & 100.0 \\
\hline Belmont & 36 & 5.8 & 43 & 7.0 & 253 & 41.1 & 339 & 55.0 & 102 & 16.6 & 72 & 11.7 & 616 & 100.0 \\
\hline John Hunter & 602 & 19.3 & 195 & 6.3 & 792 & 25.4 & 1431 & 45.9 & 455 & 14.6 & 421 & 13.5 & 3118 & 100.0 \\
\hline Christo Road Private & 367 & 31.5 & 51 & 4.4 & 197 & 16.9 & 446 & 38.3 & 266 & 22.8 & 93 & 8.0 & 1165 & 100.0 \\
\hline Manning Base & 68 & 10.7 & 46 & 7.2 & 266 & 41.9 & 352 & 55.4 & 64 & 10.1 & 84 & 13.2 & 635 & 100.0 \\
\hline Armidale & 15 & 3.4 & 27 & 6.2 & 110 & 25.1 & 236 & 53.8 & 46 & 10.5 & 44 & 10.0 & 439 & 100.0 \\
\hline Inverell & 1 & 0.5 & 3 & 1.4 & 41 & 19.5 & 99 & 47.1 & 56 & 26.7 & 42 & 20.0 & 210 & 100.0 \\
\hline Tamworth Base & 130 & 21.2 & 74 & 12.1 & 155 & 25.2 & 333 & 54.2 & 56 & 9.1 & 43 & 7.0 & 614 & 100.0 \\
\hline Other Area hospitals & 116 & 9.7 & 85 & 7.1 & 273 & 22.7 & 630 & 52.5 & 150 & 12.5 & 206 & 17.2 & 1201 & 100.0 \\
\hline ALL HOSPITALS & 1460 & 15.0 & 604 & 6.2 & 2620 & 27.0 & 4783 & 49.2 & 1535 & 15.8 & 1249 & 12.9 & 9713 & 100.0 \\
\hline
\end{tabular}


TABLE 117 (continued)

CONFINEMENTS BY TYPE OF PAIN RELIEF AND HOSPITAL, NSW 2003

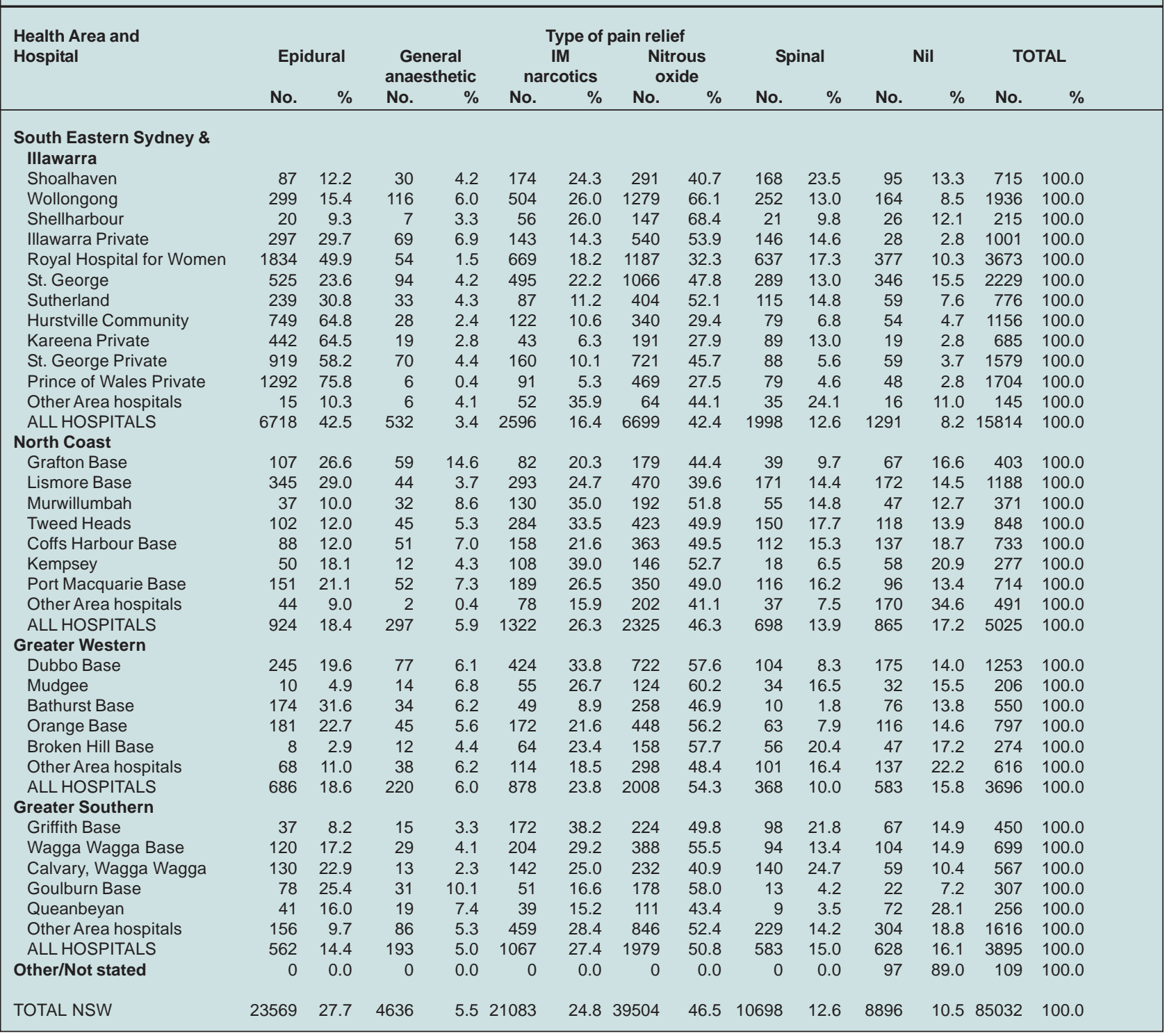

Source: NSW Midwives Data Collection (HOIST). Centre for Epidemiology and Research, NSW Department of Health.

\# Hospitals with more than 200 deliveries are identified individually. All hospitals include all public and private hospitals. 


\section{Perineal status in selected hospitals}

Table 118 show the perineal status in vaginal deliveries for individual hospitals where the number of reported confinements exceeded 200 in 2003, totals for all hospitals within each health area and the NSW total.
In addition to the perinatal outcomes described in the table there were a total of 89 cases of fourth degree tear reported in 2003.

\section{TABLE 118}

CONFINEMENTS WITH VAGINAL DELIVERIES BY PERINEAL STATUS AND HOSPITAL, NSW 2003\#

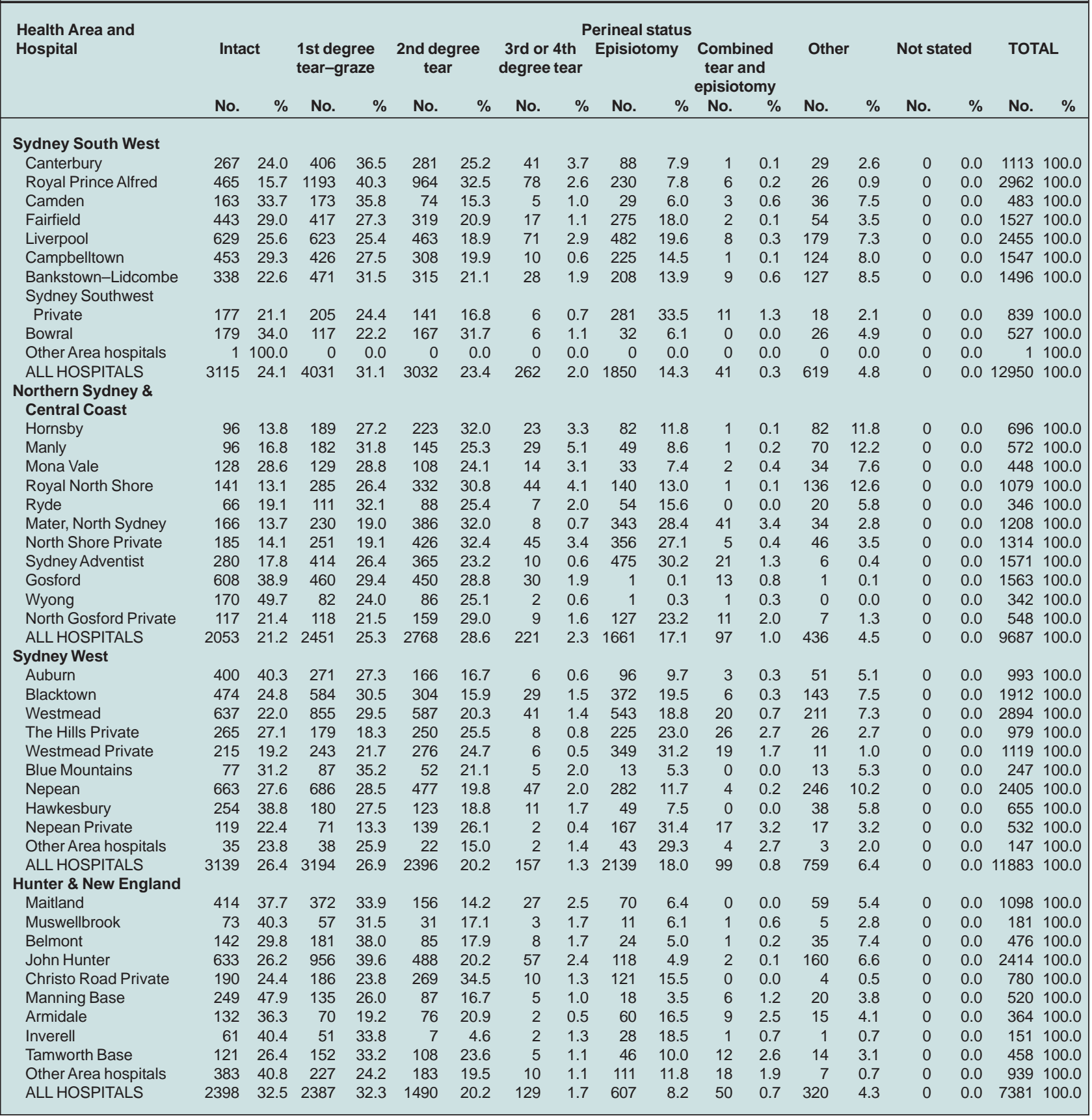


TABLE 118 (continued)

CONFINEMENTS WITH VAGINAL DELIVERIES BY PERINEAL STATUS AND HOSPITAL, NSW 2003\#

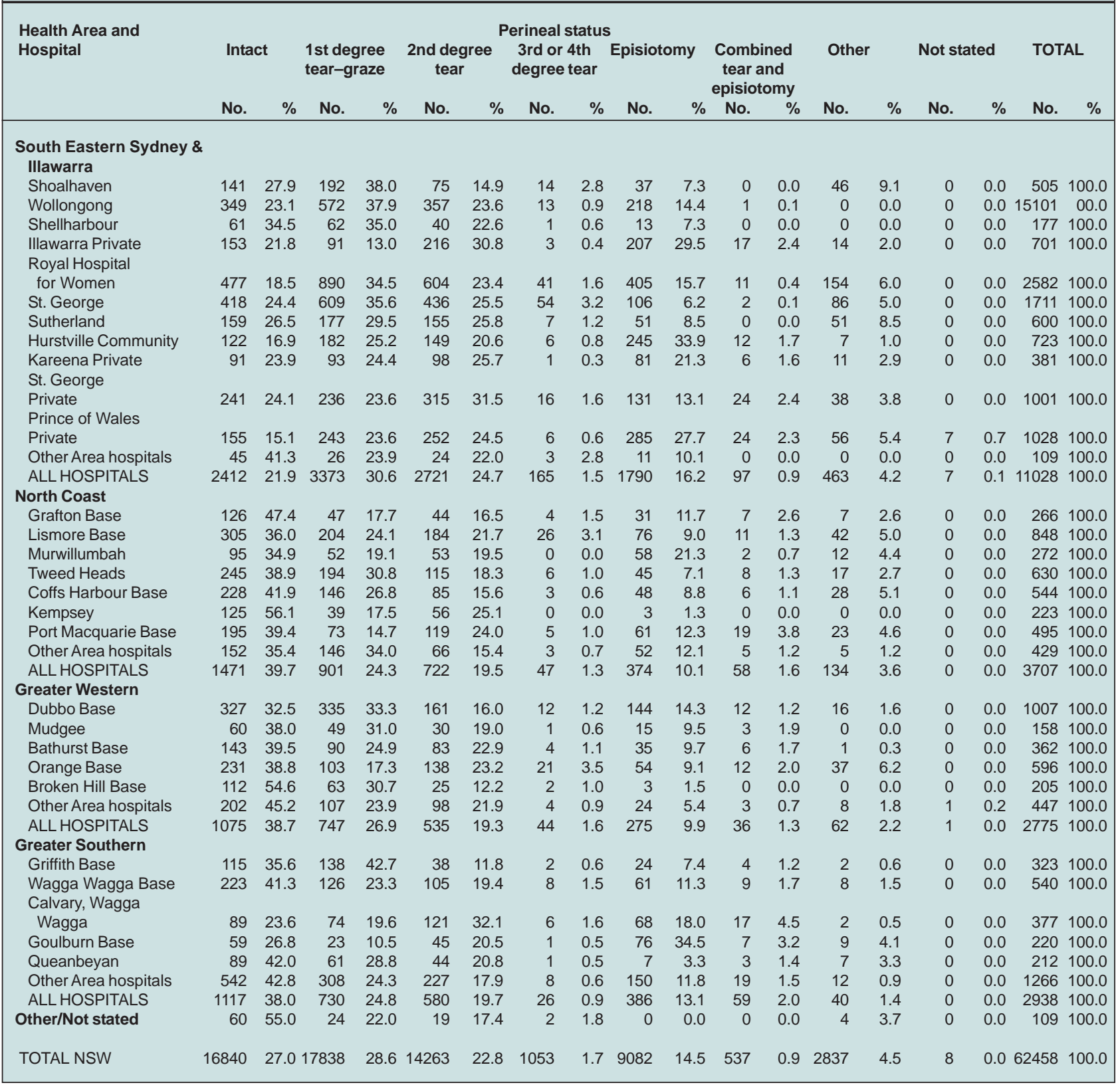

Source: NSW Midwives Data Collection (HOIST). Centre for Epidemiology and Research, NSW Department of Health.

\# Hospitals with more than 200 deliveries are identified individually. All hospitals include all public and private hospitals. 


\section{Birthweight in selected hospitals}

Table 119 shows the birthweight ofbabies for individual hospitals where the number of reported confinements exceeded 200 in 2003, totals for all hospitals within each health area and the NSW total.

\section{TABLE 119}

BIRTHS BY BIRTHWEIGHT AND HOSPITAL, NSW 2003"

\begin{tabular}{|c|c|c|c|c|c|c|c|c|c|c|c|c|}
\hline \multirow{3}{*}{$\begin{array}{l}\text { Health Area and } \\
\text { Hospital }\end{array}$} & \multicolumn{10}{|c|}{ Birthweight (grams) } & \multirow{2}{*}{\multicolumn{2}{|c|}{ Total }} \\
\hline & \multicolumn{2}{|c|}{$\begin{array}{l}\text { Less than } \\
1,000\end{array}$} & \multicolumn{2}{|c|}{$\begin{array}{c}1,000- \\
1,499\end{array}$} & \multicolumn{2}{|c|}{$\begin{array}{l}1,500- \\
2,499\end{array}$} & \multicolumn{2}{|c|}{$2,500+$} & \multicolumn{2}{|c|}{ Not stated } & & \\
\hline & No. & $\%$ & No. & $\%$ & No. & $\%$ & No. & $\%$ & No. & $\%$ & No. & $\%$ \\
\hline \multicolumn{13}{|l|}{ Sydney South West } \\
\hline Canterbury & 5 & 0.4 & 1 & 0.1 & 50 & 3.6 & 1350 & 96.0 & 0 & 0.0 & 1406 & 100.0 \\
\hline Royal Prince Alfred & 60 & 1.4 & 57 & 1.4 & 253 & 6.1 & 3771 & 91.0 & 2 & 0.0 & 4143 & 100.0 \\
\hline Camden & 0 & 0.0 & 0 & 0.0 & 2 & 0.4 & 530 & 99.4 & 1 & 0.2 & 533 & 100.0 \\
\hline Fairfield & 4 & 0.2 & 3 & 0.2 & 77 & 4.2 & 1743 & 95.4 & 1 & 0.1 & 1828 & 100.0 \\
\hline Liverpool & 56 & 1.8 & 73 & 2.3 & 268 & 8.5 & 2770 & 87.5 & 0 & 0.0 & 3167 & 100.0 \\
\hline Campbelltown & 9 & 0.4 & 3 & 0.1 & 106 & 5.2 & 1924 & 94.2 & 0 & 0.0 & 2042 & 100.0 \\
\hline Bankstown-Lidcombe & 10 & 0.5 & 0 & 0.0 & 66 & 3.6 & 1758 & 95.8 & 1 & 0.1 & 1835 & 100.0 \\
\hline Sydney Southwest Private & 0 & 0.0 & 0 & 0.0 & 44 & 3.7 & 1133 & 96.3 & 0 & 0.0 & 1177 & 100.0 \\
\hline Bowral & 2 & 0.3 & 0 & 0.0 & 18 & 2.7 & 643 & 97.0 & 0 & 0.0 & 663 & 100.0 \\
\hline Other Area hospitals & 1 & 50.0 & 0 & 0.0 & 0 & 0.0 & 1 & 50.0 & 0 & 0.0 & 2 & 100.0 \\
\hline ALL HOSPITALS & 147 & 0.9 & 137 & 0.8 & 884 & 5.3 & 15623 & 93.0 & 5 & 0.0 & 16796 & 100.0 \\
\hline \multirow{2}{*}{\multicolumn{13}{|c|}{ Northern Sydney \& }} \\
\hline & & & & & & & & & & & & \\
\hline Hornsby & 3 & 0.3 & 1 & 0.1 & 25 & 2.7 & 890 & 96.8 & 0 & 0.0 & 919 & 100.0 \\
\hline Manly & 2 & 0.3 & 1 & 0.1 & 20 & 2.5 & 767 & 97.1 & 0 & 0.0 & 790 & 100.0 \\
\hline Mona Vale & 1 & 0.2 & 1 & 0.2 & 21 & 3.6 & 568 & 96.1 & 0 & 0.0 & 591 & 100.0 \\
\hline Royal North Shore & 32 & 1.9 & 64 & 3.8 & 163 & 9.6 & 1432 & 84.6 & 2 & 0.1 & 1693 & 100.0 \\
\hline Ryde & 4 & 0.9 & 1 & 0.2 & 9 & 2.0 & 430 & 96.8 & 0 & 0.0 & 444 & 100.0 \\
\hline Mater, North Sydney & 4 & 0.2 & 3 & 0.1 & 65 & 3.1 & 2031 & 96.6 & 0 & 0.0 & 2103 & 100.0 \\
\hline North Shore Private & 12 & 0.5 & 3 & 0.1 & 72 & 3.2 & 2189 & 96.2 & 0 & 0.0 & 2276 & 100.0 \\
\hline Sydney Adventist & 10 & 0.4 & 1 & 0.0 & 73 & 3.1 & 2249 & 96.4 & 0 & 0.0 & 2333 & 100.0 \\
\hline Gosford & 6 & 0.3 & 4 & 0.2 & 109 & 5.1 & 2033 & 94.5 & 0 & 0.0 & 2152 & 100.0 \\
\hline North Gosford Private & 0 & 0.0 & 0 & 0.0 & 36 & 4.0 & 860 & 96.0 & 0 & 0.0 & 896 & 100.0 \\
\hline Wyong & 0 & 0.0 & 0 & 0.0 & 3 & 0.8 & 352 & 99.2 & 0 & 0.0 & 355 & 100.0 \\
\hline ALL HOSPITALS & 74 & 0.5 & 79 & 0.5 & 596 & 4.1 & 13801 & 94.8 & 2 & 0.0 & 14552 & 100.0 \\
\hline \multicolumn{13}{|l|}{ Sydney West } \\
\hline Auburn & 3 & 0.3 & 2 & 0.2 & 41 & 3.4 & 1153 & 96.2 & 0 & 0.0 & 1199 & 100.0 \\
\hline Blacktown & 14 & 0.6 & 12 & 0.5 & 138 & 5.5 & 2331 & 93.4 & 0 & 0.0 & 2495 & 100.0 \\
\hline Westmead & 75 & 1.9 & 75 & 1.9 & 287 & 7.2 & 3562 & 89.0 & 3 & 0.1 & 4002 & 100.0 \\
\hline The Hills Private & 2 & 0.1 & 1 & 0.1 & 44 & 3.3 & 1290 & 96.5 & 0 & 0.0 & 1337 & 100.0 \\
\hline Westmead Private & 5 & 0.3 & 3 & 0.2 & 59 & 3.7 & 1518 & 95.7 & 1 & 0.1 & 1586 & 100.0 \\
\hline Blue Mountains & 5 & 1.6 & 1 & 0.3 & 11 & 3.4 & 305 & 94.7 & 0 & 0.0 & 322 & 100.0 \\
\hline Nepean & 46 & 1.4 & 38 & 1.1 & 260 & 7.8 & 2973 & 89.5 & 3 & 0.1 & 3320 & 100.0 \\
\hline Hawkesbury & 3 & 0.3 & 0 & 0.0 & 22 & 2.5 & 853 & 97.0 & 1 & 0.1 & 879 & 100.0 \\
\hline Nepean Private & 3 & 0.3 & 0 & 0.0 & 22 & 2.5 & 856 & 97.2 & 0 & 0.0 & 881 & 100.0 \\
\hline Other Area hospitals & 0 & 0.0 & 0 & 0.0 & 6 & 2.9 & 204 & 97.1 & 0 & 0.0 & 210 & 100.0 \\
\hline ALL HOSPITALS & 156 & 1.0 & 132 & 0.8 & 890 & 5.5 & 15045 & 92.7 & 8 & 0.0 & 16231 & 100.0 \\
\hline \multicolumn{13}{|l|}{ Hunter \& New England } \\
\hline Maitland & 2 & 0.1 & 1 & 0.1 & 77 & 5.1 & 1434 & 94.7 & 0 & 0.0 & 1514 & 100.0 \\
\hline Muswellbrook & 0 & 0.0 & 0 & 0.0 & 3 & 1.3 & 223 & 98.7 & 0 & 0.0 & 226 & 100.0 \\
\hline Belmont & 1 & 0.2 & 0 & 0.0 & 20 & 3.2 & 601 & 96.6 & 0 & 0.0 & 622 & 100.0 \\
\hline John Hunter & 68 & 2.1 & 52 & 1.6 & 251 & 7.9 & 2820 & 88.3 & 1 & 0.0 & 3192 & 100.0 \\
\hline Christo Road Private & 1 & 0.1 & 1 & 0.1 & 48 & 4.1 & 1134 & 95.8 & 0 & 0.0 & 1184 & 100.0 \\
\hline Manning Base & 5 & 0.8 & 2 & 0.3 & 37 & 5.8 & 596 & 92.7 & 3 & 0.5 & 643 & 100.0 \\
\hline Armidale & 3 & 0.7 & 0 & 0.0 & 32 & 7.1 & 415 & 92.0 & 1 & 0.2 & 451 & 100.0 \\
\hline Inverell & 0 & 0.0 & 1 & 0.5 & 6 & 2.8 & 204 & 96.7 & 0 & 0.0 & 211 & 100.0 \\
\hline Tamworth Base & 4 & 0.6 & 1 & 0.2 & 58 & 9.3 & 564 & 90.0 & 0 & 0.0 & 627 & 100.0 \\
\hline Other Area hospitals & 3 & 0.2 & 1 & 0.1 & 24 & 2.0 & 1180 & 97.7 & 0 & 0.0 & 1208 & 100.0 \\
\hline ALL HOSPITALS & 87 & 0.9 & 59 & 0.6 & 556 & 5.6 & 9171 & 92.8 & 5 & 0.1 & 9878 & 100.0 \\
\hline
\end{tabular}


TABLE 119 (continued)

BIRTHS BY BIRTHWEIGHT AND HOSPITAL, NSW 2003"

\begin{tabular}{|c|c|c|c|c|c|c|c|c|c|c|c|c|}
\hline \multirow{3}{*}{$\begin{array}{l}\text { Health Area and } \\
\text { Hospital }\end{array}$} & \multicolumn{12}{|c|}{ Birthweight (grams) } \\
\hline & \multicolumn{2}{|c|}{$\begin{array}{l}\text { Less than } \\
1,000\end{array}$} & \multicolumn{2}{|c|}{$\begin{array}{c}1,000- \\
1,499\end{array}$} & \multicolumn{2}{|c|}{$\begin{array}{l}1,500- \\
2,499\end{array}$} & \multicolumn{2}{|c|}{$2,500+$} & \multicolumn{2}{|c|}{ Not stated } & \multicolumn{2}{|c|}{ Total } \\
\hline & No. & $\%$ & No. & $\%$ & No. & $\%$ & No. & $\%$ & No. & $\%$ & No. & $\%$ \\
\hline \multicolumn{13}{|l|}{$\begin{array}{l}\text { South Eastern Sydney \& } \\
\text { Illawarra }\end{array}$} \\
\hline Shoalhaven & 5 & 0.7 & 0 & 0.0 & 37 & 5.1 & 679 & 94.2 & 0 & 0.0 & 721 & 100.0 \\
\hline Wollongong & 11 & 0.6 & 10 & 0.5 & 114 & 5.8 & 1836 & 93.2 & 0 & 0.0 & 1971 & 100.0 \\
\hline Shellharbour & 0 & 0.0 & 0 & 0.0 & 4 & 1.9 & 211 & 98.1 & 0 & 0.0 & 215 & 100.0 \\
\hline Illawarra Private & 1 & 0.1 & 0 & 0.0 & 16 & 1.6 & 989 & 98.3 & 0 & 0.0 & 1006 & 100.0 \\
\hline Royal Hospital for Women & 58 & 1.5 & 41 & 1.1 & 237 & 6.3 & 3419 & 90.9 & 5 & 0.1 & 3760 & 100.0 \\
\hline St. George & 10 & 0.4 & 1 & 0.0 & 109 & 4.8 & 2135 & 94.6 & 1 & 0.0 & 2256 & 100.0 \\
\hline Sutherland & 2 & 0.3 & 1 & 0.1 & 27 & 3.4 & 756 & 95.9 & 2 & 0.3 & 788 & 100.0 \\
\hline Hurstville Community & 3 & 0.3 & 1 & 0.1 & 44 & 3.7 & 1133 & 95.9 & 0 & 0.0 & 1181 & 100.0 \\
\hline Kareena Private & 1 & 0.1 & 2 & 0.3 & 29 & 4.2 & 664 & 95.3 & 1 & 0.1 & 697 & 100.0 \\
\hline St. George Private & 3 & 0.2 & 2 & 0.1 & 70 & 4.4 & 1532 & 95.3 & 0 & 0.0 & 1607 & 100.0 \\
\hline Prince of Wales Private & 0 & 0.0 & 0 & 0.0 & 42 & 2.4 & 1668 & 96.7 & 15 & 0.9 & 1725 & 100.0 \\
\hline Other Area hospitals & 0 & 0.0 & 0 & 0.0 & 1 & 0.7 & 144 & 99.3 & 0 & 0.0 & 145 & 100.0 \\
\hline ALL HOSPITALS & 94 & 0.6 & 58 & 0.4 & 730 & 4.5 & 15166 & 94.4 & 24 & 0.1 & 16072 & 100.0 \\
\hline \multicolumn{13}{|l|}{ North Coast } \\
\hline Grafton Base & 3 & 0.7 & 0 & 0.0 & 21 & 5.2 & 381 & 94.1 & 0 & 0.0 & 405 & 100.0 \\
\hline Lismore Base & 7 & 0.6 & 6 & 0.5 & 70 & 5.8 & 1122 & 93.0 & 2 & 0.2 & 1207 & 100.0 \\
\hline Murwillumbah & 1 & 0.3 & 1 & 0.3 & 16 & 4.2 & 360 & 95.2 & 0 & 0.0 & 378 & 100.0 \\
\hline Tweed Heads & 9 & 1.0 & 1 & 0.1 & 49 & 5.7 & 799 & 93.1 & 0 & 0.0 & 858 & 100.0 \\
\hline Coffs Harbour Base & 6 & 0.8 & 3 & 0.4 & 42 & 5.6 & 694 & 93.0 & 1 & 0.1 & 746 & 100.0 \\
\hline Kempsey & 0 & 0.0 & 1 & 0.4 & 12 & 4.3 & 264 & 95.3 & 0 & 0.0 & 277 & 100.0 \\
\hline Port Macquarie Base & 3 & 0.4 & 1 & 0.1 & 33 & 4.6 & 686 & 94.9 & 0 & 0.0 & 723 & 100.0 \\
\hline Other Area hospitals & 1 & 0.2 & 0 & 0.0 & 13 & 2.6 & 478 & 97.0 & 1 & 0.2 & 493 & 100.0 \\
\hline ALL HOSPITALS & 30 & 0.6 & 13 & 0.3 & 256 & 5.0 & 4784 & 94.0 & 4 & 0.1 & 5087 & 100.0 \\
\hline \multicolumn{13}{|l|}{ Greater Western } \\
\hline Dubbo Base & 4 & 0.3 & 6 & 0.5 & 68 & 5.3 & 1194 & 93.7 & 2 & 0.2 & 1274 & 100.0 \\
\hline Mudgee & 0 & 0.0 & 0 & 0.0 & 6 & 2.9 & 200 & 97.1 & 0 & 0.0 & 206 & 100.0 \\
\hline Bathurst Base & 2 & 0.4 & 1 & 0.2 & 26 & 4.7 & 530 & 94.8 & 0 & 0.0 & 559 & 100.0 \\
\hline Orange Base & 5 & 0.6 & 2 & 0.2 & 55 & 6.8 & 752 & 92.4 & 0 & 0.0 & 814 & 100.0 \\
\hline Broken Hill Base & 1 & 0.4 & 0 & 0.0 & 14 & 5.1 & 262 & 94.6 & 0 & 0.0 & 277 & 100.0 \\
\hline Other Area hospitals & 1 & 0.2 & 1 & 0.2 & 23 & 3.7 & 591 & 95.9 & 0 & 0.0 & 616 & 100.0 \\
\hline ALL HOSPITALS & 13 & 0.3 & 10 & 0.3 & 192 & 5.1 & 3529 & 94.2 & 2 & 0.1 & 3746 & 100.0 \\
\hline \multicolumn{13}{|l|}{ Greater Southern } \\
\hline Griffith Base & 2 & 0.4 & 2 & 0.4 & 21 & 4.6 & 431 & 94.5 & 0 & 0.0 & 456 & 100.0 \\
\hline Wagga Wagga Base & 5 & 0.7 & 2 & 0.3 & 51 & 7.1 & 659 & 91.9 & 0 & 0.0 & 717 & 100.0 \\
\hline Calvary, Wagga Wagga & 0 & 0.0 & 1 & 0.2 & 28 & 4.8 & 550 & 95.0 & 0 & 0.0 & 579 & 100.0 \\
\hline Goulburn Base & 4 & 1.3 & 2 & 0.6 & 8 & 2.6 & 296 & 95.5 & 0 & 0.0 & 310 & 100.0 \\
\hline Queanbeyan & 1 & 0.4 & 0 & 0.0 & 4 & 1.6 & 251 & 98.0 & 0 & 0.0 & 256 & 100.0 \\
\hline Other Area hospitals & 3 & 0.2 & 2 & 0.1 & 52 & 3.2 & 1566 & 96.4 & 2 & 0.1 & 1625 & 100.0 \\
\hline ALL HOSPITALS & 15 & 0.4 & 9 & 0.2 & 164 & 4.2 & 3753 & 95.2 & 2 & 0.1 & 3943 & 100.0 \\
\hline Other/Not stated & 0 & 0.0 & 0 & 0.0 & 2 & 1.8 & 107 & 98.2 & 0 & 0.0 & 109 & 100.0 \\
\hline TOTAL NSW & 616 & 0.7 & 497 & 0.6 & 4270 & 4.9 & 80979 & 93.7 & 52 & 0.1 & 86414 & 100.0 \\
\hline
\end{tabular}

Source: NSW Midwives Data Collection (HOIST). Centre for Epidemiology and Research, NSW Department of Health.

\# Hospitals with more than 200 total deliveries are identified individually. All hospitals include all public and private hospitals. 


\section{Gestational age in selected hospitals}

Table 120 shows the gestational age of babies for individual hospitals where the number of reported confinements exceeded 200 in 2003, totals for all hospitals within each health area and the NSW total.

\section{TABLE 120}

BIRTHS BY GESTATIONAL AGE AND HOSPITAL, NSW 2003\#

\begin{tabular}{|c|c|c|c|c|c|c|c|c|c|c|c|c|}
\hline \multirow{3}{*}{$\begin{array}{l}\text { Health Area and } \\
\text { Hospital }\end{array}$} & \multicolumn{12}{|c|}{ Gestational age (weeks) } \\
\hline & \multicolumn{2}{|c|}{ Less than 31} & \multicolumn{2}{|c|}{$32-33$} & \multicolumn{2}{|c|}{$34-36$} & \multicolumn{2}{|c|}{$37+$} & \multicolumn{2}{|c|}{ Not stated } & \multicolumn{2}{|c|}{ TOTAL } \\
\hline & No. & $\%$ & No. & $\%$ & No. & $\%$ & No. & $\%$ & No. & $\%$ & No. & $\%$ \\
\hline \multicolumn{13}{|l|}{ Sydney South West } \\
\hline Canterbury & 5 & 0.4 & 0 & 0.0 & 52 & 3.7 & 1349 & 95.9 & 0 & 0.0 & 1406 & 100.0 \\
\hline Royal Prince Alfred & 145 & 3.5 & 75 & 1.8 & 241 & 5.8 & 3682 & 88.9 & 0 & 0.0 & 4143 & 100.0 \\
\hline Camden & 0 & 0.0 & 0 & 0.0 & 7 & 1.3 & 526 & 98.7 & 0 & 0.0 & 533 & 100.0 \\
\hline Fairfield & 6 & 0.3 & 2 & 0.1 & 66 & 3.6 & 1754 & 96.0 & 0 & 0.0 & 1828 & 100.0 \\
\hline Liverpool & 139 & 4.4 & 86 & 2.7 & 190 & 6.0 & 2752 & 86.9 & 0 & 0.0 & 3167 & 100.0 \\
\hline Campbelltown & 13 & 0.6 & 5 & 0.2 & 86 & 4.2 & 1938 & 94.9 & 0 & 0.0 & 2042 & 100.0 \\
\hline Bankstown-Lidcombe & 10 & 0.5 & 3 & 0.2 & 68 & 3.7 & 1754 & 95.6 & 0 & 0.0 & 1835 & 100.0 \\
\hline Bowral & 2 & 0.3 & 1 & 0.2 & 14 & 2.1 & 646 & 97.4 & 0 & 0.0 & 663 & 100.0 \\
\hline Sydney Southwest Private & 1 & 0.1 & 1 & 0.1 & 66 & 5.6 & 1109 & 94.2 & 0 & 0.0 & 1177 & 100.0 \\
\hline Other Area hospitals & 1 & 50.0 & 0 & 0.0 & 0 & 0.0 & 1 & 50.0 & 0 & 0.0 & 2 & 100.0 \\
\hline ALL HOSPITALS & 322 & 1.9 & 173 & 1.0 & 790 & 4.7 & 15511 & 92.3 & 0 & 0.0 & 16796 & 100.0 \\
\hline \multicolumn{13}{|l|}{ Northern Sydney \& } \\
\hline Hornsby & 3 & 0.3 & 0 & 0.0 & 33 & 3.6 & 883 & 96.1 & 0 & 0.0 & 919 & 100.0 \\
\hline Manly & 3 & 0.4 & 0 & 0.0 & 23 & 2.9 & 764 & 96.7 & 0 & 0.0 & 790 & 100.0 \\
\hline Mona Vale & 1 & 0.2 & 1 & 0.2 & 25 & 4.2 & 564 & 95.4 & 0 & 0.0 & 591 & 100.0 \\
\hline Royal North Shore & 109 & 6.4 & 64 & 3.8 & 83 & 4.9 & 1437 & 84.9 & 0 & 0.0 & 1693 & 100.0 \\
\hline Ryde & 5 & 1.1 & 1 & 0.2 & 7 & 1.6 & 431 & 97.1 & 0 & 0.0 & 444 & 100.0 \\
\hline Mater, North Sydney & 7 & 0.3 & 5 & 0.2 & 102 & 4.9 & 1989 & 94.6 & 0 & 0.0 & 2103 & 100.0 \\
\hline North Shore Private & 15 & 0.7 & 15 & 0.7 & 85 & 3.7 & 2161 & 94.9 & 0 & 0.0 & 2276 & 100.0 \\
\hline Sydney Adventist & 11 & 0.5 & 6 & 0.3 & 87 & 3.7 & 2229 & 95.5 & 0 & 0.0 & 2333 & 100.0 \\
\hline Gosford & 12 & 0.6 & 15 & 0.7 & 168 & 7.8 & 1957 & 90.9 & 0 & 0.0 & 2152 & 100.0 \\
\hline North Gosford Private & 1 & 0.1 & 3 & 0.3 & 45 & 5.0 & 847 & 94.5 & 0 & 0.0 & 896 & 100.0 \\
\hline Wyong & 0 & 0.0 & 1 & 0.3 & 5 & 1.4 & 349 & 98.3 & 0 & 0.0 & 355 & 100.0 \\
\hline ALL HOSPITALS & 167 & 1.1 & 111 & 0.8 & 663 & 4.6 & 13611 & 93.5 & 0 & 0.0 & 14552 & 100.0 \\
\hline \multicolumn{13}{|l|}{ Sydney West } \\
\hline Auburn & 6 & 0.5 & 3 & 0.3 & 29 & 2.4 & 1161 & 96.8 & 0 & 0.0 & 1199 & 100.0 \\
\hline Blacktown & 24 & 1.0 & 13 & 0.5 & 110 & 4.4 & 2348 & 94.1 & 0 & 0.0 & 2495 & 100.0 \\
\hline Westmead & 154 & 3.8 & 71 & 1.8 & 212 & 5.3 & 3565 & 89.1 & 0 & 0.0 & 4002 & 100.0 \\
\hline The Hills Private & 4 & 0.3 & 1 & 0.1 & 56 & 4.2 & 1276 & 95.4 & 0 & 0.0 & 1337 & 100.0 \\
\hline Westmead Private & 9 & 0.6 & 3 & 0.2 & 72 & 4.5 & 1502 & 94.7 & 0 & 0.0 & 1586 & 100.0 \\
\hline Blue Mountains & 5 & 1.6 & 3 & 0.9 & 6 & 1.9 & 308 & 95.7 & 0 & 0.0 & 322 & 100.0 \\
\hline Nepean & 86 & 2.6 & 71 & 2.1 & 184 & 5.5 & 2979 & 89.7 & 0 & 0.0 & 3320 & 100.0 \\
\hline Hawkesbury & 5 & 0.6 & 0 & 0.0 & 36 & 4.1 & 838 & 95.3 & 0 & 0.0 & 879 & 100.0 \\
\hline Nepean Private & 3 & 0.3 & 1 & 0.1 & 28 & 3.2 & 849 & 96.4 & 0 & 0.0 & 881 & 100.0 \\
\hline Other Area hospitals & 0 & 0.0 & 0 & 0.0 & 7 & 3.3 & 203 & 96.7 & 0 & 0.0 & 210 & 100.0 \\
\hline ALL HOSPITALS & 296 & 1.8 & 166 & 1.0 & 740 & 4.6 & 15029 & 92.6 & 0 & 0.0 & 16231 & 100.0 \\
\hline \multicolumn{13}{|l|}{ Hunter \& New England } \\
\hline Maitland & 4 & 0.3 & 11 & 0.7 & 85 & 5.6 & 1414 & 93.4 & 0 & 0.0 & 1514 & 100.0 \\
\hline Muswellbrook & 0 & 0.0 & 0 & 0.0 & 4 & 1.8 & 221 & 97.8 & 1 & 0.4 & 226 & 100.0 \\
\hline Belmont & 1 & 0.2 & 0 & 0.0 & 31 & 5.0 & 590 & 94.9 & 0 & 0.0 & 622 & 100.0 \\
\hline John Hunter & 131 & 4.1 & 74 & 2.3 & 203 & 6.4 & 2784 & 87.2 & 0 & 0.0 & 3192 & 100.0 \\
\hline Christo Road Private & 1 & 0.1 & 4 & 0.3 & 73 & 6.2 & 1106 & 93.4 & 0 & 0.0 & 1184 & 100.0 \\
\hline Manning Base & 13 & 2.0 & 3 & 0.5 & 31 & 4.8 & 596 & 92.7 & 0 & 0.0 & 643 & 100.0 \\
\hline Armidale & 4 & 0.9 & 0 & 0.0 & 27 & 6.0 & 420 & 93.1 & 0 & 0.0 & 451 & 100.0 \\
\hline Inverell & 1 & 0.5 & 0 & 0.0 & 6 & 2.8 & 204 & 96.7 & 0 & 0.0 & 211 & 100.0 \\
\hline Tamworth Base & 6 & 1.0 & 5 & 0.8 & 64 & 10.2 & 552 & 88.0 & 0 & 0.0 & 627 & 100.0 \\
\hline Other Area hospitals & 6 & 0.5 & 4 & 0.3 & 28 & 2.3 & 1170 & 96.9 & 0 & 0.0 & 1208 & 100.0 \\
\hline ALL HOSPITALS & 167 & 1.7 & 101 & 1.0 & 552 & 5.6 & 9057 & 91.7 & 1 & 0.0 & 9878 & 100.0 \\
\hline
\end{tabular}


TABLE 120 (continued)

BIRTHS BY GESTATIONAL AGE AND HOSPITAL, NSW 2003\#

\begin{tabular}{|c|c|c|c|c|c|c|c|c|c|c|c|c|}
\hline \multirow{3}{*}{$\begin{array}{l}\text { Health Area and } \\
\text { Hospital }\end{array}$} & \multicolumn{12}{|c|}{ Gestational age (weeks) } \\
\hline & \multicolumn{2}{|c|}{ Less than 31} & \multicolumn{2}{|c|}{$32-33$} & \multicolumn{2}{|c|}{$34-36$} & \multicolumn{2}{|c|}{$37+$} & \multicolumn{2}{|c|}{ Not stated } & \multicolumn{2}{|c|}{ TOTAL } \\
\hline & No. & $\%$ & No. & $\%$ & No. & $\%$ & No. & $\%$ & No. & $\%$ & No. & $\%$ \\
\hline \multicolumn{13}{|l|}{$\begin{array}{l}\text { South Eastern Sydney \& } \\
\text { Illawarra }\end{array}$} \\
\hline Shoalhaven & 5 & 0.7 & 3 & 0.4 & 31 & 4.3 & 682 & 94.6 & 0 & 0.0 & 721 & 100.0 \\
\hline Wollongong & 19 & 1.0 & 25 & 1.3 & 142 & 7.2 & 1784 & 90.5 & 1 & 0.1 & 1971 & 100.0 \\
\hline Shellharbour & 0 & 0.0 & 1 & 0.5 & 3 & 1.4 & 211 & 98.1 & 0 & 0.0 & 215 & 100.0 \\
\hline Illawarra Private & 1 & 0.1 & 0 & 0.0 & 17 & 1.7 & 988 & 98.2 & 0 & 0.0 & 1006 & 100.0 \\
\hline Royal Hospital for Women & 111 & 3.0 & 54 & 1.4 & 176 & 4.7 & 3419 & 90.9 & 0 & 0.0 & 3760 & 100.0 \\
\hline St. George & 13 & 0.6 & 12 & 0.5 & 107 & 4.7 & 2124 & 94.1 & 0 & 0.0 & 2256 & 100.0 \\
\hline Sutherland & 6 & 0.8 & 5 & 0.6 & 31 & 3.9 & 746 & 94.7 & 0 & 0.0 & 788 & 100.0 \\
\hline Hurstville Community & 4 & 0.3 & 1 & 0.1 & 49 & 4.1 & 1127 & 95.4 & 0 & 0.0 & 1181 & 100.0 \\
\hline Kareena Private & 1 & 0.1 & 10 & 1.4 & 38 & 5.5 & 648 & 93.0 & 0 & 0.0 & 697 & 100.0 \\
\hline St. George Private & 5 & 0.3 & 4 & 0.2 & 62 & 3.9 & 1536 & 95.6 & 0 & 0.0 & 1607 & 100.0 \\
\hline Prince of Wales Private & 2 & 0.1 & 1 & 0.1 & 51 & 3.0 & 1663 & 96.4 & 8 & 0.5 & 1725 & 100.0 \\
\hline Other Area hospitals & 0 & 0.0 & 0 & 0.0 & 1 & 0.7 & 144 & 99.3 & 0 & 0.0 & 145 & 100.0 \\
\hline ALL HOSPITALS & 167 & 1.0 & 116 & 0.7 & 708 & 4.4 & 15072 & 93.8 & 9 & 0.1 & 16072 & 100.0 \\
\hline \multicolumn{13}{|l|}{ North Coast } \\
\hline Grafton Base & 3 & 0.7 & 0 & 0.0 & 21 & 5.2 & 381 & 94.1 & 0 & 0.0 & 405 & 100.0 \\
\hline Lismore Base & 13 & 1.1 & 9 & 0.7 & 73 & 6.0 & 1112 & 92.1 & 0 & 0.0 & 1207 & 100.0 \\
\hline Murwillumbah & 1 & 0.3 & 3 & 0.8 & 16 & 4.2 & 358 & 94.7 & 0 & 0.0 & 378 & 100.0 \\
\hline Tweed Heads & 10 & 1.2 & 3 & 0.3 & 57 & 6.6 & 788 & 91.8 & 0 & 0.0 & 858 & 100.0 \\
\hline Coffs Harbour Base & 10 & 1.3 & 4 & 0.5 & 44 & 5.9 & 688 & 92.2 & 0 & 0.0 & 746 & 100.0 \\
\hline Kempsey & 2 & 0.7 & 0 & 0.0 & 11 & 4.0 & 264 & 95.3 & 0 & 0.0 & 277 & 100.0 \\
\hline Port Macquarie Base & 4 & 0.6 & 6 & 0.8 & 47 & 6.5 & 666 & 92.1 & 0 & 0.0 & 723 & 100.0 \\
\hline Other Area hospitals & 1 & 0.2 & 0 & 0.0 & 12 & 2.4 & 480 & 97.4 & 0 & 0.0 & 493 & 100.0 \\
\hline ALL HOSPITALS & 44 & 0.9 & 25 & 0.5 & 281 & 5.5 & 4737 & 93.1 & 0 & 0.0 & 5087 & 100.0 \\
\hline \multicolumn{13}{|l|}{ Greater Western } \\
\hline Dubbo Base & 11 & 0.9 & 9 & 0.7 & 73 & 5.7 & 1181 & 92.7 & 0 & 0.0 & 1274 & 100.0 \\
\hline Mudgee & 0 & 0.0 & 0 & 0.0 & 5 & 2.4 & 201 & 97.6 & 0 & 0.0 & 206 & 100.0 \\
\hline Bathurst Base & 4 & 0.7 & 5 & 0.9 & 21 & 3.8 & 529 & 94.6 & 0 & 0.0 & 559 & 100.0 \\
\hline Orange Base & 10 & 1.2 & 2 & 0.2 & 63 & 7.7 & 739 & 90.8 & 0 & 0.0 & 814 & 100.0 \\
\hline Broken Hill Base & 1 & 0.4 & 0 & 0.0 & 20 & 7.2 & 256 & 92.4 & 0 & 0.0 & 277 & 100.0 \\
\hline Other Area hospitals & 4 & 0.6 & 3 & 0.5 & 9 & 1.5 & 600 & 97.4 & 0 & 0.0 & 616 & 100.0 \\
\hline ALL HOSPITALS & 30 & 0.8 & 19 & 0.5 & 191 & 5.1 & 3506 & 93.6 & 0 & 0.0 & 3746 & 100.0 \\
\hline \multicolumn{13}{|l|}{ Greater Southern } \\
\hline Griffith Base & 5 & 1.1 & 1 & 0.2 & 16 & 3.5 & 434 & 95.2 & 0 & 0.0 & 456 & 100.0 \\
\hline Wagga Wagga Base & 8 & 1.1 & 11 & 1.5 & 44 & 6.1 & 654 & 91.2 & 0 & 0.0 & 717 & 100.0 \\
\hline Calvary, Wagga Wagga & 4 & 0.7 & 4 & 0.7 & 33 & 5.7 & 538 & 92.9 & 0 & 0.0 & 579 & 100.0 \\
\hline Goulburn Base & 6 & 1.9 & 1 & 0.3 & 11 & 3.5 & 292 & 94.2 & 0 & 0.0 & 310 & 100.0 \\
\hline Queanbeyan & 1 & 0.4 & 0 & 0.0 & 2 & 0.8 & 253 & 98.8 & 0 & 0.0 & 256 & 100.0 \\
\hline Other Area hospitals & 8 & 0.5 & 2 & 0.1 & 49 & 3.0 & 1566 & 96.4 & 0 & 0.0 & 1625 & 100.0 \\
\hline ALL HOSPITALS & 32 & 0.8 & 19 & 0.5 & 155 & 3.9 & 3737 & 94.8 & 0 & 0.0 & 3943 & 100.0 \\
\hline Other/Not stated & 0 & 0.0 & 0 & 0.0 & 0 & 0.0 & 109 & 100.0 & 0 & 0.0 & 109 & 100.0 \\
\hline TOTAL NSW & 1225 & 1.4 & 730 & 0.8 & 4080 & 4.7 & 80369 & 93.0 & 10 & 0.0 & 86414 & 100.0 \\
\hline
\end{tabular}

Source: NSW Midwives Data Collection (HOIST). Centre for Epidemiology and Research, NSW Department of Health.

\# Hospitals with more than 200 deliveries are identified individually. All hospitals include all public and private hospitals. 


\section{Admission to special care and neonatal intensive care units in selected hospitals}

Table 121 shows admissions of liveborn babies to special care and neonatal intensive care units for individual hospitals where the number of reported confinements exceeded 200 in 2003, totals for all hospitals within each health area and the NSW total. The number of babies admitted to neonatal intensive care units reported here is higher than the numbers reported in Chapter 8 , as some babies admitted to a neonatal intensive care unit do not meet the registration criteria for inclusion in the Neonatal Intensive Care Units Data Collection.

\section{TABLE 121}

BIRTHS BY ADMISSION TO SPECIAL CARE OR NEONATAL INTENSIVE CARE UNIT AND HOSPITAL, NSW 2003\#

\begin{tabular}{|c|c|c|c|c|c|c|c|c|c|c|c|c|c|c|c|c|}
\hline \multirow{3}{*}{$\begin{array}{l}\text { Health Area and } \\
\text { Hospital }\end{array}$} & \multicolumn{8}{|c|}{ Admission to special care unit } & \multicolumn{8}{|c|}{ Admission to neonatal intensive care unit } \\
\hline & \multicolumn{2}{|c|}{ No } & \multicolumn{2}{|c|}{ Yes } & \multicolumn{2}{|c|}{ Not stated } & \multicolumn{2}{|c|}{ TOTAL } & \multicolumn{2}{|c|}{ No } & \multicolumn{2}{|c|}{ Yes } & \multicolumn{2}{|c|}{ Not stated } & \multicolumn{2}{|c|}{ TOTAL } \\
\hline & No. & $\%$ & No. & $\%$ & No. & $\%$ & No. & $\%$ & No. & $\%$ & No. & $\%$ & No. & $\%$ & No. & $\%$ \\
\hline \multicolumn{17}{|l|}{ Sydney South West } \\
\hline Canterbury & 1194 & 85.5 & 202 & 14.5 & 0 & 0.0 & 1396 & 100.0 & 1396 & 100.0 & 0 & 0.0 & 0 & 0.0 & 1396 & 100.0 \\
\hline Royal Prince Alfred & 3770 & 91.7 & 343 & 8.3 & 0 & 0.0 & 4113 & 100.0 & 3881 & 94.4 & 232 & 5.6 & 0 & 0.0 & 4113 & 100.0 \\
\hline Camden & 514 & 96.8 & 17 & 3.2 & 0 & 0.0 & 531 & 100.0 & 528 & 99.4 & 3 & 0.6 & 0 & 0.0 & 531 & 100.0 \\
\hline Fairfield & 1400 & 76.9 & 421 & 23.1 & 0 & 0.0 & 1821 & 100.0 & 1814 & 99.6 & 7 & 0.4 & 0 & 0.0 & 1821 & 100.0 \\
\hline Liverpool & 2762 & 87.9 & 380 & 12.1 & 0 & 0.0 & 3142 & 100.0 & 2993 & 95.3 & 149 & 4.7 & 0 & 0.0 & 3142 & 100.0 \\
\hline Campbelltown & 1715 & 84.5 & 315 & 15.5 & 0 & 0.0 & 2030 & 100.0 & 2019 & 99.5 & 11 & 0.5 & 0 & 0.0 & 2030 & 100.0 \\
\hline Bankstown-Lidcombe & e 1513 & 82.9 & 312 & 17.1 & 0 & 0.0 & 1825 & 100.0 & 1811 & 99.2 & 14 & 0.8 & 0 & 0.0 & 1825 & 100.0 \\
\hline \multicolumn{17}{|l|}{ Sydney Southwest } \\
\hline Private & 915 & 77.8 & 261 & 22.2 & 0 & 0.0 & 1176 & 100.0 & 1168 & 99.3 & 8 & 0.7 & 0 & 0.0 & 1176 & 100.0 \\
\hline Bowral & 571 & 86.5 & 89 & 13.5 & 0 & 0.0 & 660 & 100.0 & 657 & 99.5 & 3 & 0.5 & 0 & 0.0 & 660 & 100.0 \\
\hline Other Area hospitals & 0 & 0.0 & 1 & 100.0 & 0 & 0.0 & 1 & 100.0 & 1 & 100.0 & 0 & 0.0 & 0 & 0.0 & 1 & 100.0 \\
\hline ALL HOSPITALS & 14354 & 86.0 & 2341 & 14.0 & 0 & 0.0 & 16695 & 100.0 & 16268 & 97.4 & 427 & 2.6 & 0 & 0.0 & 16695 & 100.0 \\
\hline \multicolumn{17}{|l|}{$\begin{array}{c}\text { Northern Sydney \& } \\
\text { Central Coast }\end{array}$} \\
\hline Hornsby & 496 & 54.1 & 421 & 45.9 & 0 & 0.0 & 917 & 100.0 & 912 & 99.5 & 5 & 0.5 & 0 & 0.0 & 917 & 100.0 \\
\hline Manly & 639 & 81.2 & 148 & 18.8 & 0 & 0.0 & 787 & 100.0 & 787 & 100.0 & 0 & 0.0 & 0 & 0.0 & 787 & 100.0 \\
\hline Mona Vale & 461 & 78.3 & 128 & 21.7 & 0 & 0.0 & 589 & 100.0 & 589 & 100.0 & 0 & 0.0 & 0 & 0.0 & 589 & 100.0 \\
\hline Royal North Shore & 1550 & 92.2 & 131 & 7.8 & 0 & 0.0 & 1681 & 100.0 & 1431 & 85.1 & 250 & 14.9 & 0 & 0.0 & 1681 & 100.0 \\
\hline Ryde & 363 & 82.5 & 77 & 17.5 & 0 & 0.0 & 440 & 100.0 & 440 & 100.0 & 0 & 0.0 & 0 & 0.0 & 440 & 100.0 \\
\hline Mater, North Sydney & 1848 & 88.0 & 252 & 12.0 & 0 & 0.0 & 2100 & 100.0 & 2078 & 99.0 & 22 & 1.0 & 0 & 0.0 & 2100 & 100.0 \\
\hline North Shore Private & 2103 & 93.1 & 155 & 6.9 & 0 & 0.0 & 2258 & 100.0 & 2252 & 99.7 & 6 & 0.3 & 0 & 0.0 & 2258 & 100.0 \\
\hline Sydney Adventist & 1978 & 85.3 & 342 & 14.7 & 0 & 0.0 & 2320 & 100.0 & 2313 & 99.7 & 7 & 0.3 & 0 & 0.0 & 2320 & 100.0 \\
\hline Gosford & 1897 & 88.5 & 247 & 11.5 & 0 & 0.0 & 2144 & 100.0 & 2123 & 99.0 & 21 & 1.0 & 0 & 0.0 & 2144 & 100.0 \\
\hline Wyong & 346 & 97.5 & 9 & 2.5 & 0 & 0.0 & 355 & 100.0 & 354 & 99.7 & 1 & 0.3 & 0 & 0.0 & 355 & 100.0 \\
\hline North Gosford Private & 770 & 86.0 & 125 & 14.0 & 0 & 0.0 & 895 & 100.0 & 889 & 99.3 & 6 & 0.7 & 0 & 0.0 & 895 & 100.0 \\
\hline ALL HOSPITALS & 12451 & 86.0 & 2035 & 14.0 & 0 & 0.0 & 14486 & 100.0 & 14168 & 97.8 & 318 & 2.2 & 0 & 0.0 & 14486 & 100.0 \\
\hline Sydney West & & & & & & & & & & & & & & & & \\
\hline Auburn & 852 & 71.7 & 337 & 28.3 & 0 & 0.0 & 1189 & 100.0 & 1188 & 99.9 & 1 & 0.1 & 0 & 0.0 & 1189 & 100.0 \\
\hline Blacktown & 2065 & 83.3 & 415 & 16.7 & 0 & 0.0 & 2480 & 100.0 & 2476 & 99.8 & 4 & 0.2 & 0 & 0.0 & 2480 & 100.0 \\
\hline Westmead & 3495 & 88.3 & 465 & 11.7 & 0 & 0.0 & 3960 & 100.0 & 3513 & 88.7 & 447 & 11.3 & 0 & 0.0 & 3960 & 100.0 \\
\hline The Hills Private & 1065 & 79.8 & 269 & 20.2 & 0 & 0.0 & 1334 & 100.0 & 1326 & 99.4 & 8 & 0.6 & 0 & 0.0 & 1334 & 100.0 \\
\hline Westmead Private & 1243 & 78.7 & 337 & 21.3 & 0 & 0.0 & 1580 & 100.0 & 1577 & 99.8 & 3 & 0.2 & 0 & 0.0 & 1580 & 100.0 \\
\hline Blue Mountains & 284 & 88.8 & 36 & 11.3 & 0 & 0.0 & 320 & 100.0 & 318 & 99.4 & 2 & 0.6 & 0 & 0.0 & 320 & 100.0 \\
\hline Nepean & 2820 & 85.6 & 473 & 14.4 & 0 & 0.0 & 3293 & 100.0 & 2903 & 88.2 & 390 & 11.8 & 0 & 0.0 & 3293 & 100.0 \\
\hline Hawkesbury & 749 & 85.7 & 125 & 14.3 & 0 & 0.0 & 874 & 100.0 & 874 & 100.0 & 0 & 0.0 & 0 & 0.0 & 874 & 100.0 \\
\hline Nepean Private & 733 & 83.8 & 142 & 16.2 & 0 & 0.0 & 875 & 100.0 & 872 & 99.7 & 3 & 0.3 & 0 & 0.0 & 875 & 100.0 \\
\hline Other Area hospitals & 200 & 95.2 & 10 & 4.8 & 0 & 0.0 & 210 & 100.0 & 209 & 99.5 & 1 & 0.5 & 0 & 0.0 & 210 & 100.0 \\
\hline ALL HOSPITALS & 13506 & 83.8 & 2609 & 16.2 & 0 & 0.0 & 16115 & 100.0 & 15256 & 94.7 & 859 & 5.3 & 0 & 0.0 & 16115 & 100.0 \\
\hline Hunter \& New Englanc & & & & & & & & & & & & & & & & \\
\hline Maitland & 1250 & 82.9 & 258 & 17.1 & 0 & 0.0 & 1508 & 100.0 & 1504 & 99.7 & 4 & 0.3 & 0 & 0.0 & 1508 & 100.0 \\
\hline Muswellbrook & 221 & 97.8 & 5 & 2.2 & 0 & 0.0 & 226 & 100.0 & 225 & 99.6 & 1 & 0.4 & 0 & 0.0 & 226 & 100.0 \\
\hline Belmont & 543 & 87.4 & 78 & 12.6 & 0 & 0.0 & 621 & 100.0 & 620 & 99.8 & 1 & 0.2 & 0 & 0.0 & 621 & 100.0 \\
\hline John Hunter & 2688 & 85.4 & 458 & 14.6 & 0 & 0.0 & 3146 & 100.0 & 2941 & 93.5 & 205 & 6.5 & 0 & 0.0 & 3146 & 100.0 \\
\hline Christo Road Private & 986 & 83.5 & 195 & 16.5 & 0 & 0.0 & 1181 & 100.0 & 1180 & 99.9 & 1 & 0.1 & 0 & 0.0 & 1181 & 100.0 \\
\hline Manning Base & 555 & 87.3 & 81 & 12.7 & 0 & 0.0 & 636 & 100.0 & 632 & 99.4 & 4 & 0.6 & 0 & 0.0 & 636 & 100.0 \\
\hline Armidale & 369 & 82.6 & 78 & 17.4 & 0 & 0.0 & 447 & 100.0 & 447 & 100.0 & 0 & 0.0 & 0 & 0.0 & 447 & 100.0 \\
\hline Inverell & 204 & 96.7 & 7 & 3.3 & 0 & 0.0 & 211 & 100.0 & 209 & 99.1 & 2 & 0.9 & 0 & 0.0 & 211 & 100.0 \\
\hline Tamworth Base & 379 & 60.9 & 243 & 39.1 & 0 & 0.0 & 622 & 100.0 & 615 & 98.9 & 7 & 1.1 & 0 & 0.0 & 622 & 100.0 \\
\hline Other Area hospitals & 1140 & 94.7 & 64 & 5.3 & 0 & 0.0 & 1204 & 100.0 & 1196 & 99.3 & 8 & 0.7 & 0 & 0.0 & 1204 & 100.0 \\
\hline ALL HOSPITALS & 8335 & 85.0 & 1467 & 15.0 & 0 & 0.0 & 9802 & 100.0 & 9569 & 97.6 & 233 & 2.4 & 0 & 0.0 & 9802 & 100.0 \\
\hline
\end{tabular}


TABLE 121 (continued)

BIRTHS BY ADMISSION TO SPECIAL CARE OR NEONATAL INTENSIVE CARE UNIT AND HOSPITAL, NSW 2003\#

\begin{tabular}{|c|c|c|c|c|c|c|c|c|c|c|c|c|c|c|c|c|}
\hline \multirow{3}{*}{$\begin{array}{l}\text { Health Area and } \\
\text { Hospital }\end{array}$} & \multicolumn{8}{|c|}{ Admission to special care unit } & \multicolumn{8}{|c|}{ Admission to neonatal intensive care unit } \\
\hline & \multicolumn{2}{|c|}{ No } & \multicolumn{2}{|c|}{ Yes } & \multicolumn{2}{|c|}{ Not stated } & \multicolumn{2}{|c|}{ TOTAL } & \multicolumn{2}{|c|}{ No } & \multicolumn{2}{|c|}{ Yes } & \multicolumn{2}{|c|}{ Not stated } & \multicolumn{2}{|c|}{ TOTAL } \\
\hline & No. & $\%$ & No. & $\%$ & No. & $\%$ & No. & $\%$ & No. & $\%$ & No. & $\%$ & No. & $\%$ & No. & $\%$ \\
\hline \multicolumn{17}{|c|}{$\begin{array}{l}\text { South Eastern Sydney \& } \\
\text { Illawarra }\end{array}$} \\
\hline Shoalhaven & 610 & 85.4 & 104 & 14.6 & 0 & 0.0 & 714 & 100.0 & 713 & 99.9 & 1 & 0.1 & 0 & 0.0 & 714 & 100.0 \\
\hline Wollongong & 1574 & 80.2 & 389 & 19.8 & 0 & 0.0 & 1963 & 100.0 & 1936 & 98.6 & 27 & 1.4 & 0 & 0.0 & 1963 & 100.0 \\
\hline Shellharbour & 201 & 93.9 & 13 & 6.1 & 0 & 0.0 & 214 & 100.0 & 213 & 99.5 & 1 & 0.5 & 0 & 0.0 & 214 & 100.0 \\
\hline Illawarra Private & 904 & 90.0 & 100 & 10.0 & 0 & 0.0 & 1004 & 100.0 & 999 & 99.5 & 5 & 0.5 & 0 & 0.0 & 1004 & 100.0 \\
\hline \multicolumn{17}{|l|}{ Royal Hospital for } \\
\hline Women & 3263 & 87.5 & 465 & 12.5 & 0 & 0.0 & 3728 & 100.0 & 3523 & 94.5 & 205 & 5.5 & 0 & 0.0 & 3728 & 100.0 \\
\hline St. George & 1922 & 85.7 & 320 & 14.3 & 0 & 0.0 & 2242 & 100.0 & 2235 & 99.7 & 7 & 0.3 & 0 & 0.0 & 2242 & 100.0 \\
\hline Sutherland & 654 & 83.3 & 131 & 16.7 & 0 & 0.0 & 785 & 100.0 & 782 & 99.6 & 3 & 0.4 & 0 & 0.0 & 785 & 100.0 \\
\hline Hurstville Community & 952 & 80.7 & 227 & 19.3 & 0 & 0.0 & 1179 & 100.0 & 1172 & 99.4 & 7 & 0.6 & 0 & 0.0 & 1179 & 100.0 \\
\hline Kareena Private & 532 & 76.4 & 164 & 23.6 & 0 & 0.0 & 696 & 100.0 & 687 & 98.7 & 9 & 1.3 & 0 & 0.0 & 696 & 100.0 \\
\hline $\begin{array}{l}\text { St. George Private } \\
\text { Prince of Wales }\end{array}$ & 1305 & 81.5 & 296 & 18.5 & 0 & 0.0 & 1601 & 100.0 & 1597 & 99.8 & 4 & 0.2 & 0 & 0.0 & 1601 & 100.0 \\
\hline Private & 1395 & 81.0 & 256 & 14.9 & 72 & 4.2 & 1723 & 100.0 & 1710 & 99.2 & 8 & 0.5 & 5 & 0.3 & 1723 & 100.0 \\
\hline Other Area hospitals & 143 & 98.6 & 2 & 1.4 & 0 & 0.0 & 145 & 100.0 & 145 & 100.0 & 0 & 0.0 & 0 & 0.0 & 145 & 100.0 \\
\hline ALL HOSPITALS & 13455 & 84.1 & 2467 & 15.4 & 72 & 0.5 & 15994 & 100.0 & 15712 & 98.2 & 277 & 1.7 & 5 & 0.0 & 15994 & 100.0 \\
\hline \multicolumn{17}{|l|}{ North Coast } \\
\hline Grafton Base & 336 & 83.4 & 67 & 16.6 & 0 & 0.0 & 403 & 100.0 & 394 & 97.8 & 9 & 2.2 & 0 & 0.0 & 403 & 100.0 \\
\hline Lismore Base & 904 & 75.8 & 289 & 24.2 & 0 & 0.0 & 1193 & 100.0 & 1182 & 99.1 & 11 & 0.9 & 0 & 0.0 & 1193 & 100.0 \\
\hline Murwillumbah & 333 & 88.3 & 44 & 11.7 & 0 & 0.0 & 377 & 100.0 & 373 & 98.9 & 4 & 1.1 & 0 & 0.0 & 377 & 100.0 \\
\hline Tweed Heads & 667 & 78.6 & 182 & 21.4 & 0 & 0.0 & 849 & 100.0 & 844 & 99.4 & 5 & 0.6 & 0 & 0.0 & 849 & 100.0 \\
\hline Coffs Harbour Base & 627 & 84.7 & 113 & 15.3 & 0 & 0.0 & 740 & 100.0 & 726 & 98.1 & 14 & 1.9 & 0 & 0.0 & 740 & 100.0 \\
\hline Kempsey & 261 & 94.9 & 14 & 5.1 & 0 & 0.0 & 275 & 100.0 & 275 & 100.0 & 0 & 0.0 & 0 & 0.0 & 275 & 100.0 \\
\hline Port Macquarie Base & 571 & 79.6 & 146 & 20.4 & 0 & 0.0 & 717 & 100.0 & 707 & 98.6 & 10 & 1.4 & 0 & 0.0 & 717 & 100.0 \\
\hline Other Area hospitals & 468 & 95.1 & 24 & 4.9 & 0 & 0.0 & 492 & 100.0 & 491 & 99.8 & 1 & 0.2 & 0 & 0.0 & 492 & 100.0 \\
\hline ALL HOSPITALS & 4167 & 82.6 & 879 & 17.4 & 0 & 0.0 & 5046 & 100.0 & 4992 & 98.9 & 54 & 1.1 & 0 & 0.0 & 5046 & 100.0 \\
\hline \multicolumn{17}{|l|}{ Greater Western } \\
\hline Dubbo Base & 1038 & 82.6 & 219 & 17.4 & 0 & 0.0 & 1257 & 100.0 & 1238 & 98.5 & 19 & 1.5 & 0 & 0.0 & 1257 & 100.0 \\
\hline Mudgee & 197 & 95.6 & 9 & 4.4 & 0 & 0.0 & 206 & 100.0 & 205 & 99.5 & 1 & 0.5 & 0 & 0.0 & 206 & 100.0 \\
\hline Bathurst Base & 476 & 85.3 & 82 & 14.7 & 0 & 0.0 & 558 & 100.0 & 544 & 97.5 & 14 & 2.5 & 0 & 0.0 & 558 & 100.0 \\
\hline Orange Base & 665 & 82.1 & 145 & 17.9 & 0 & 0.0 & 810 & 100.0 & 795 & 98.1 & 15 & 1.9 & 0 & 0.0 & 810 & 100.0 \\
\hline Broken Hill Base & 249 & 90.5 & 26 & 9.5 & 0 & 0.0 & 275 & 100.0 & 271 & 98.5 & 4 & 1.5 & 0 & 0.0 & 275 & 100.0 \\
\hline Other Area hospitals & 571 & 92.8 & 44 & 7.2 & 0 & 0.0 & 615 & 100.0 & 610 & 99.2 & 5 & 0.8 & 0 & 0.0 & 615 & 100.0 \\
\hline ALL HOSPITALS & 3196 & 85.9 & 525 & 14.1 & 0 & 0.0 & 3721 & 100.0 & 3663 & 98.4 & 58 & 1.6 & 0 & 0.0 & 3721 & 100.0 \\
\hline \multicolumn{17}{|l|}{ Greater Southern } \\
\hline Wagga Wagga Base & 579 & 81.2 & 134 & 18.8 & 0 & 0.0 & 713 & 100.0 & 712 & 99.9 & 1 & 0.1 & 0 & 0.0 & 713 & 100.0 \\
\hline Griffith Base & 259 & 57.2 & 194 & 42.8 & 0 & 0.0 & 453 & 100.0 & 450 & 99.3 & 3 & 0.7 & 0 & 0.0 & 453 & 100.0 \\
\hline \multicolumn{17}{|l|}{ Calvary, Wagga } \\
\hline Wagga & 508 & 87.7 & 71 & 12.3 & 0 & 0.0 & 579 & 100.0 & 574 & 99.1 & 5 & 0.9 & 0 & 0.0 & 579 & 100.0 \\
\hline Goulburn Base & 256 & 84.5 & 47 & 15.5 & 0 & 0.0 & 303 & 100.0 & 295 & 97.4 & 8 & 2.6 & 0 & 0.0 & 303 & 100.0 \\
\hline Queanbeyan & 243 & 94.9 & 13 & 5.1 & 0 & 0.0 & 256 & 100.0 & 250 & 97.7 & 6 & 2.3 & 0 & 0.0 & 256 & 100.0 \\
\hline Other Area hospitals & 1476 & 91.2 & 142 & 8.8 & 0 & 0.0 & 1618 & 100.0 & 1592 & 98.4 & 26 & 1.6 & 0 & 0.0 & 1618 & 100.0 \\
\hline ALL HOSPITALS & 3321 & 84.7 & 601 & 15.3 & 0 & 0.0 & 3922 & 100.0 & 3873 & 98.8 & 49 & 1.2 & 0 & 0.0 & 3922 & 100.0 \\
\hline Other/Not stated & 108 & 99.1 & 1 & 0.9 & 0 & 0.0 & 109 & 100.0 & 107 & 98.2 & 2 & 1.8 & 0 & 0.0 & 109 & 100.0 \\
\hline TOTAL NSW & 72893 & 84.9 & 12925 & 15.0 & 72 & 0.1 & 85890 & 100.0 & 83608 & 97.3 & 2277 & 2.7 & 5 & 0.0 & 85890 & 100.0 \\
\hline
\end{tabular}

Source: NSW Midwives Data Collection (HOIST). Centre for Epidemiology and Research, NSW Department of Health.

\# Hospitals with more than 200 deliveries are identified individually. All hospitals include all public and private hospitals. 


\section{Baby discharge status in selected hospitals}

Table 122 shows the discharge status of babies born in hospitals where the number of reported confinements exceeded 200 in 2003, totals for all hospitals within each health area and the NSW total.

\section{TABLE 122}

BIRTHS BY BABY DISCHARGE STATUS AND HOSPITAL, NSW 2003\#

\begin{tabular}{|c|c|c|c|c|c|c|c|c|c|c|c|c|}
\hline \multirow{3}{*}{$\begin{array}{l}\text { Health Area and } \\
\text { Hospital }\end{array}$} & \multicolumn{12}{|c|}{ Baby discharge status } \\
\hline & \multicolumn{2}{|c|}{ Discharged } & \multicolumn{2}{|c|}{ Stillborn } & \multicolumn{2}{|c|}{$\begin{array}{l}\text { Neonatal } \\
\text { death }\end{array}$} & \multicolumn{2}{|c|}{ Transferred } & \multicolumn{2}{|c|}{ Not stated } & \multicolumn{2}{|c|}{ TOTAL } \\
\hline & No. & $\%$ & No. & $\%$ & No. & $\%$ & No. & $\%$ & No. & $\%$ & No. & $\%$ \\
\hline \multicolumn{13}{|l|}{ Sydney South West } \\
\hline Canterbury & 1380 & 98.2 & 10 & 0.7 & 2 & 0.1 & 14 & 1.0 & 0 & 0.0 & 1406 & 100.0 \\
\hline Royal Prince Alfred & 3962 & 95.6 & 30 & 0.7 & 20 & 0.5 & 131 & 3.2 & 0 & 0.0 & 4143 & 100.0 \\
\hline Camden & 521 & 97.7 & 2 & 0.4 & 0 & 0.0 & 10 & 1.9 & 0 & 0.0 & 533 & 100.0 \\
\hline Fairfield & 1799 & 98.4 & 7 & 0.4 & 4 & 0.2 & 18 & 1.0 & 0 & 0.0 & 1828 & 100.0 \\
\hline Liverpool & 2945 & 93.0 & 25 & 0.8 & 19 & 0.6 & 178 & 5.6 & 0 & 0.0 & 3167 & 100.0 \\
\hline Campbelltown & 1994 & 97.6 & 12 & 0.6 & 3 & 0.1 & 33 & 1.6 & 0 & 0.0 & 2042 & 100.0 \\
\hline Bankstown-Lidcombe & 1804 & 98.3 & 10 & 0.5 & 6 & 0.3 & 15 & 0.8 & 0 & 0.0 & 1835 & 100.0 \\
\hline Sydney Southwest Private & 1159 & 98.5 & 1 & 0.1 & 0 & 0.0 & 17 & 1.4 & 0 & 0.0 & 1177 & 100.0 \\
\hline Bowral & 470 & 70.9 & 3 & 0.5 & 0 & 0.0 & 190 & 28.7 & 0 & 0.0 & 663 & 100.0 \\
\hline Other Area hospitals & 1 & 50.0 & 1 & 50.0 & 0 & 0.0 & 0 & 0.0 & 0 & 0.0 & 2 & 100.0 \\
\hline ALL HOSPITALS & 16035 & 95.5 & 101 & 0.6 & 54 & 0.3 & 606 & 3.6 & 0 & 0.0 & 16796 & 100.0 \\
\hline \multirow{2}{*}{\multicolumn{13}{|c|}{ Northern Sydney \& }} \\
\hline & & & & & & & & & & & & \\
\hline Hornsby & 907 & 98.7 & 2 & 0.2 & 2 & 0.2 & 8 & 0.9 & 0 & 0.0 & 919 & 100.0 \\
\hline Manly & 776 & 98.2 & 3 & 0.4 & 1 & 0.1 & 10 & 1.3 & 0 & 0.0 & 790 & 100.0 \\
\hline Mona Vale & 583 & 98.6 & 2 & 0.3 & 0 & 0.0 & 6 & 1.0 & 0 & 0.0 & 591 & 100.0 \\
\hline Royal North Shore & 1522 & 89.9 & 12 & 0.7 & 10 & 0.6 & 149 & 8.8 & 0 & 0.0 & 1693 & 100.0 \\
\hline Ryde & 434 & 97.7 & 4 & 0.9 & 1 & 0.2 & 5 & 1.1 & 0 & 0.0 & 444 & 100.0 \\
\hline Mater, North Sydney & 2072 & 98.5 & 3 & 0.1 & 3 & 0.1 & 25 & 1.2 & 0 & 0.0 & 2103 & 100.0 \\
\hline North Shore Private & 2247 & 98.7 & 18 & 0.8 & 5 & 0.2 & 6 & 0.3 & 0 & 0.0 & 2276 & 100.0 \\
\hline Sydney Adventist & 2311 & 99.1 & 13 & 0.6 & 1 & 0.0 & 8 & 0.3 & 0 & 0.0 & 2333 & 100.0 \\
\hline Gosford & 1866 & 86.7 & 8 & 0.4 & 0 & 0.0 & 278 & 12.9 & 0 & 0.0 & 2152 & 100.0 \\
\hline North Gosford Private & 888 & 99.1 & 1 & 0.1 & 0 & 0.0 & 7 & 0.8 & 0 & 0.0 & 896 & 100.0 \\
\hline Wyong & 344 & 96.9 & 0 & 0.0 & 0 & 0.0 & 11 & 3.1 & 0 & 0.0 & 355 & 100.0 \\
\hline ALL HOSPITALS & 13950 & 95.9 & 66 & 0.5 & 23 & 0.2 & 513 & 3.5 & 0 & 0.0 & 14552 & 100.0 \\
\hline \multicolumn{13}{|l|}{ Sydney West } \\
\hline Auburn & 1177 & 98.2 & 10 & 0.8 & 0 & 0.0 & 12 & 1.0 & 0 & 0.0 & 1199 & 100.0 \\
\hline Blacktown & 2449 & 98.2 & 15 & 0.6 & 5 & 0.2 & 26 & 1.0 & 0 & 0.0 & 2495 & 100.0 \\
\hline Westmead & 3703 & 92.5 & 42 & 1.0 & 21 & 0.5 & 236 & 5.9 & 0 & 0.0 & 4002 & 100.0 \\
\hline The Hills Private & 1322 & 98.9 & 3 & 0.2 & 0 & 0.0 & 12 & 0.9 & 0 & 0.0 & 1337 & 100.0 \\
\hline Westmead Private & 1572 & 99.1 & 6 & 0.4 & 2 & 0.1 & 6 & 0.4 & 0 & 0.0 & 1586 & 100.0 \\
\hline Blue Mountains & 307 & 95.3 & 2 & 0.6 & 1 & 0.3 & 12 & 3.7 & 0 & 0.0 & 322 & 100.0 \\
\hline Nepean & 3141 & 94.6 & 27 & 0.8 & 10 & 0.3 & 142 & 4.3 & 0 & 0.0 & 3320 & 100.0 \\
\hline Hawkesbury & 858 & 97.6 & 5 & 0.6 & 1 & 0.1 & 15 & 1.7 & 0 & 0.0 & 879 & 100.0 \\
\hline Nepean Private & 873 & 99.1 & 6 & 0.7 & 0 & 0.0 & 2 & 0.2 & 0 & 0.0 & 881 & 100.0 \\
\hline Other Area hospitals & 207 & 98.6 & 0 & 0.0 & 0 & 0.0 & 3 & 1.4 & 0 & 0.0 & 210 & 100.0 \\
\hline ALL HOSPITALS & 15609 & 96.2 & 116 & 0.7 & 40 & 0.2 & 466 & 2.9 & 0 & 0.0 & 16231 & 100.0 \\
\hline \multicolumn{13}{|l|}{ Hunter \& New England } \\
\hline Maitland & 1213 & 80.1 & 6 & 0.4 & 0 & 0.0 & 295 & 19.5 & 0 & 0.0 & 1514 & 100.0 \\
\hline Muswellbrook & 222 & 98.2 & 0 & 0.0 & 0 & 0.0 & 4 & 1.8 & 0 & 0.0 & 226 & 100.0 \\
\hline Belmont & 592 & 95.2 & 1 & 0.2 & 0 & 0.0 & 29 & 4.7 & 0 & 0.0 & 622 & 100.0 \\
\hline John Hunter & 2597 & 81.4 & 46 & 1.4 & 32 & 1.0 & 517 & 16.2 & 0 & 0.0 & 3192 & 100.0 \\
\hline Christo Road Private & 1108 & 93.6 & 3 & 0.3 & 1 & 0.1 & 72 & 6.1 & 0 & 0.0 & 1184 & 100.0 \\
\hline Manning Base & 609 & 94.7 & 7 & 1.1 & 2 & 0.3 & 25 & 3.9 & 0 & 0.0 & 643 & 100.0 \\
\hline Armidale & 423 & 93.8 & 4 & 0.9 & 1 & 0.2 & 23 & 5.1 & 0 & 0.0 & 451 & 100.0 \\
\hline Inverell & 203 & 96.2 & 0 & 0.0 & 0 & 0.0 & 8 & 3.8 & 0 & 0.0 & 211 & 100.0 \\
\hline Tamworth Base & 541 & 86.3 & 5 & 0.8 & 0 & 0.0 & 81 & 12.9 & 0 & 0.0 & 627 & 100.0 \\
\hline Other Area hospitals & 1150 & 95.2 & 4 & 0.3 & 2 & 0.2 & 52 & 4.3 & 0 & 0.0 & 1208 & 100.0 \\
\hline ALL HOSPITALS & 8658 & 87.6 & 76 & 0.8 & 38 & 0.4 & 1106 & 11.2 & 0 & 0.0 & 9878 & 100.0 \\
\hline
\end{tabular}


TABLE 122 (continued)

BIRTHS BY BABY DISCHARGE STATUS AND HOSPITAL, NSW 2003\#

\begin{tabular}{|c|c|c|c|c|c|c|c|c|c|c|c|c|}
\hline \multirow{3}{*}{$\begin{array}{l}\text { Health Area and } \\
\text { Hospital }\end{array}$} & \multicolumn{12}{|c|}{ Baby discharge status } \\
\hline & \multicolumn{2}{|c|}{ Discharged } & \multicolumn{2}{|c|}{ Stillborn } & \multicolumn{2}{|c|}{$\begin{array}{l}\text { Neonatal } \\
\text { death }\end{array}$} & \multicolumn{2}{|c|}{ Transferred } & \multicolumn{2}{|c|}{ Not stated } & \multicolumn{2}{|c|}{ TOTAL } \\
\hline & No. & $\%$ & No. & $\%$ & No. & $\%$ & No. & $\%$ & No. & $\%$ & No. & $\%$ \\
\hline \multicolumn{13}{|l|}{$\begin{array}{l}\text { South Eastern Sydney \& } \\
\text { Illawarra }\end{array}$} \\
\hline Shoalhaven & 614 & 85.2 & 7 & 1.0 & 4 & 0.6 & 96 & 13.3 & 0 & 0.0 & 721 & 100.0 \\
\hline Wollongong & 1543 & 78.3 & 8 & 0.4 & 7 & 0.4 & 413 & 21.0 & 0 & 0.0 & 1971 & 100.0 \\
\hline Shellharbour & 202 & 94.0 & 1 & 0.5 & 0 & 0.0 & 12 & 5.6 & 0 & 0.0 & 215 & 100.0 \\
\hline Illawarra Private & 987 & 98.1 & 2 & 0.2 & 0 & 0.0 & 17 & 1.7 & 0 & 0.0 & 1006 & 100.0 \\
\hline Royal Hospital for Women & 3621 & 96.3 & 32 & 0.9 & 31 & 0.8 & 76 & 2.0 & 0 & 0.0 & 3760 & 100.0 \\
\hline St. George & 2226 & 98.7 & 14 & 0.6 & 3 & 0.1 & 13 & 0.6 & 0 & 0.0 & 2256 & 100.0 \\
\hline Sutherland & 778 & 98.7 & 3 & 0.4 & 1 & 0.1 & 6 & 0.8 & 0 & 0.0 & 788 & 100.0 \\
\hline Hurstville Community & 1170 & 99.1 & 2 & 0.2 & 1 & 0.1 & 8 & 0.7 & 0 & 0.0 & 1181 & 100.0 \\
\hline Kareena Private & 681 & 97.7 & 1 & 0.1 & 0 & 0.0 & 15 & 2.2 & 0 & 0.0 & 697 & 100.0 \\
\hline St. George Private & 1589 & 98.9 & 6 & 0.4 & 0 & 0.0 & 12 & 0.7 & 0 & 0.0 & 1607 & 100.0 \\
\hline Prince of Wales Private & 1714 & 99.4 & 1 & 0.1 & 0 & 0.0 & 9 & 0.5 & 1 & 0.1 & 1725 & 100.0 \\
\hline Other Area hospitals & 143 & 98.6 & 0 & 0.0 & 0 & 0.0 & 2 & 1.4 & 0 & 0.0 & 145 & 100.0 \\
\hline ALL HOSPITALS & 15268 & 95.0 & 77 & 0.5 & 47 & 0.3 & 679 & 4.2 & 1 & 0.0 & 16072 & 100.0 \\
\hline \multicolumn{13}{|l|}{ North Coast } \\
\hline Grafton Base & 389 & 96.0 & 2 & 0.5 & 1 & 0.2 & 13 & 3.2 & 0 & 0.0 & 405 & 100.0 \\
\hline Lismore Base & 1001 & 82.9 & 14 & 1.2 & 1 & 0.1 & 191 & 15.8 & 0 & 0.0 & 1207 & 100.0 \\
\hline Murwillumbah & 369 & 97.6 & 1 & 0.3 & 1 & 0.3 & 7 & 1.9 & 0 & 0.0 & 378 & 100.0 \\
\hline Tweed Heads & 831 & 96.9 & 9 & 1.0 & 2 & 0.2 & 16 & 1.9 & 0 & 0.0 & 858 & 100.0 \\
\hline Coffs Harbour Base & 680 & 91.2 & 6 & 0.8 & 0 & 0.0 & 60 & 8.0 & 0 & 0.0 & 746 & 100.0 \\
\hline Kempsey & 267 & 96.4 & 2 & 0.7 & 0 & 0.0 & 8 & 2.9 & 0 & 0.0 & 277 & 100.0 \\
\hline Port Macquarie Base & 689 & 95.3 & 6 & 0.8 & 1 & 0.1 & 27 & 3.7 & 0 & 0.0 & 723 & 100.0 \\
\hline Other Area hospitals & 470 & 95.3 & 1 & 0.2 & 2 & 0.4 & 20 & 4.1 & 0 & 0.0 & 493 & 100.0 \\
\hline ALL HOSPITALS & 4696 & 92.3 & 41 & 0.8 & 8 & 0.2 & 342 & 6.7 & 0 & 0.0 & 5087 & 100.0 \\
\hline \multicolumn{13}{|l|}{ Greater Western } \\
\hline Dubbo Base & 821 & 64.4 & 17 & 1.3 & 0 & 0.0 & 436 & 34.2 & 0 & 0.0 & 1274 & 100.0 \\
\hline Mudgee & 199 & 96.6 & 0 & 0.0 & 1 & 0.5 & 6 & 2.9 & 0 & 0.0 & 206 & 100.0 \\
\hline Bathurst Base & 421 & 75.3 & 1 & 0.2 & 1 & 0.2 & 136 & 24.3 & 0 & 0.0 & 559 & 100.0 \\
\hline Orange Base & 670 & 82.3 & 4 & 0.5 & 2 & 0.2 & 138 & 17.0 & 0 & 0.0 & 814 & 100.0 \\
\hline Broken Hill Base & 266 & 96.0 & 2 & 0.7 & 1 & 0.4 & 8 & 2.9 & 0 & 0.0 & 277 & 100.0 \\
\hline Other Area hospitals & 569 & 92.4 & 1 & 0.2 & 1 & 0.2 & 45 & 7.3 & 0 & 0.0 & 616 & 100.0 \\
\hline ALL HOSPITALS & 2946 & 78.6 & 25 & 0.7 & 6 & 0.2 & 769 & 20.5 & 0 & 0.0 & 3746 & 100.0 \\
\hline \multicolumn{13}{|l|}{ Greater Southern } \\
\hline Griffith Base & 442 & 96.9 & 3 & 0.7 & 1 & 0.2 & 10 & 2.2 & 0 & 0.0 & 456 & 100.0 \\
\hline Wagga Wagga Base & 644 & 89.8 & 4 & 0.6 & 2 & 0.3 & 67 & 9.3 & 0 & 0.0 & 717 & 100.0 \\
\hline Calvary, Wagga Wagga & 569 & 98.3 & 0 & 0.0 & 0 & 0.0 & 10 & 1.7 & 0 & 0.0 & 579 & 100.0 \\
\hline Goulburn Base & 288 & 92.9 & 7 & 2.3 & 0 & 0.0 & 15 & 4.8 & 0 & 0.0 & 310 & 100.0 \\
\hline Queanbeyan & 243 & 94.9 & 0 & 0.0 & 1 & 0.4 & 12 & 4.7 & 0 & 0.0 & 256 & 100.0 \\
\hline Other Area hospitals & 1552 & 95.5 & 7 & 0.4 & 0 & 0.0 & 66 & 4.1 & 0 & 0.0 & 1625 & 100.0 \\
\hline ALL HOSPITALS & 3738 & 94.8 & 21 & 0.5 & 4 & 0.1 & 180 & 4.6 & 0 & 0.0 & 3943 & 100.0 \\
\hline Other/Not stated & 106 & 97.2 & 0 & 0.0 & 0 & 0.0 & 3 & 2.8 & 0 & 0.0 & 109 & 100.0 \\
\hline TOTAL NSW & 81006 & 93.7 & 523 & 0.6 & 220 & 0.3 & 4664 & 5.4 & 1 & 0.0 & 86414 & 100.0 \\
\hline
\end{tabular}

Source: NSW Midwives Data Collection (HOIST). Centre for Epidemiology and Research, NSW Department of Health.

\# Hospitals with more than 200 deliveries are identified individually. All hospitals include all public and private hospitals. 


\section{Postnatal length of stay in selected hospitals}

Table 123 shows the mother's postnatal length of stay in the hospital of birth for hospitals where the number of reported confinements exceeded 200 in 2002, totals for all hospitals within each health area and the NSW total.

\section{TABLE 123}

AVERAGE MATERNAL POSTNATAL LENGTH OF STAY IN HOSPITAL OF BIRTH, NSW 1998-2002*

\begin{tabular}{|c|c|c|c|c|c|c|c|c|c|c|c|}
\hline \multirow{2}{*}{$\begin{array}{l}\text { Health Area and } \\
\text { Hospital }\end{array}$} & \multicolumn{5}{|c|}{ Average postnatal length of stay (days) } & \multirow{2}{*}{$\begin{array}{l}\text { Health Area and } \\
\text { Hospital }\end{array}$} & \multicolumn{5}{|c|}{ iverage postnatal length of stay (days) } \\
\hline & 1998 & 1999 & 2000 & 2001 & 2002 & & 1998 & 1999 & 2000 & 2001 & 2002 \\
\hline \multicolumn{6}{|l|}{ Sydney South West } & \multicolumn{6}{|l|}{ South Eastern Sydney \& } \\
\hline Canterbury & 2.8 & 2.9 & 2.8 & 2.7 & 2.9 & Illawarra & & & & & \\
\hline Royal Prince Alfred & 3.9 & 4.0 & 3.6 & 3.7 & 3.8 & Shoalhaven & 2.5 & 2.7 & 2.6 & 2.5 & 2.3 \\
\hline Fairfield & 2.9 & 2.8 & 2.6 & 2.6 & 2.5 & Wollongong & 2.6 & 2.8 & 2.2 & 2.6 & 2.6 \\
\hline Liverpool & 2.9 & 3.0 & 2.8 & 2.7 & 2.6 & Shellharbour & 3.0 & 2.8 & 2.7 & 2.8 & 2.8 \\
\hline Campbelltown & 2.6 & 2.6 & 2.5 & 2.5 & 2.6 & Illawarra Private & 5.6 & 5.6 & 5.6 & 5.4 & 5.5 \\
\hline Bankstown-Lidcombe & 2.8 & 2.9 & 2.8 & 2.8 & 2.7 & Royal Hospital for Women & 3.8 & 3.6 & 3.5 & 3.6 & 3.6 \\
\hline Sydney Southwest Private & - & 4.5 & 4.9 & 4.6 & 4.3 & St. George & 3.6 & 3.5 & 3.3 & 2.9 & 2.7 \\
\hline Bowral & 3.0 & 3.0 & 2.7 & 2.6 & 2.4 & Sutherland & 3.8 & 3.6 & 3.2 & 3.0 & 3.1 \\
\hline ALL HOSPITALS & 3.3 & 3.2 & 3.0 & 3.0 & 3.0 & Hurstville Community & 6.4 & 5.5 & 4.5 & 4.4 & 4.5 \\
\hline \multicolumn{6}{|l|}{ Northern Sydney \& } & Kareena Private & 5.9 & 5.9 & 5.7 & 5.2 & 5.0 \\
\hline \multicolumn{6}{|l|}{ Central Coast } & St. George Private & 5.5 & 5.3 & 5.1 & 5.2 & 4.9 \\
\hline Hornsby & 3.8 & 3.7 & 3.6 & 3.5 & 3.1 & Prince of Wales Private & 5.6 & 5.2 & 4.9 & 4.6 & 4.8 \\
\hline Manly & 3.7 & 3.8 & 3.8 & 3.6 & 3.5 & ALL HOSPITALS & 4.1 & 3.9 & 3.7 & 3.8 & 3.7 \\
\hline Mona Vale & 3.8 & 3.7 & 3.8 & 3.5 & 3.5 & North Coast & & & & & \\
\hline Royal North Shore & 4.1 & 4.3 & 3.9 & 3.7 & 3.6 & Grafton Base & 3.9 & 3.9 & 3.8 & 3.5 & 3.6 \\
\hline Ryde & 3.3 & 3.4 & 3.3 & 3.1 & 3.4 & Lismore Base & 3.4 & 3.1 & 3.2 & 3.1 & 3.2 \\
\hline Mater, North Sydney & 5.2 & 5.4 & 5.2 & 4.8 & 4.5 & Murwillumbah & 3.7 & 3.7 & 3.7 & 3.6 & 3.6 \\
\hline North Shore Private & 4.8 & 4.8 & 4.7 & 4.6 & 4.5 & Coffs Harbour Base & 4.0 & 3.9 & 4.0 & 3.8 & 3.8 \\
\hline Sydney Adventist & 5.3 & 5.5 & 5.2 & 4.7 & 4.6 & Kempsey & 3.9 & 3.8 & 3.6 & 3.2 & 3.0 \\
\hline Gosford & 2.4 & 2.5 & 2.4 & 2.3 & 2.5 & Tweed Heads & 3.1 & 3.4 & 3.0 & 3.0 & 3.1 \\
\hline Wyong & 2.5 & 2.4 & 2.3 & 2.2 & 2.1 & Port Macquarie Base & 3.8 & 4.1 & 3.8 & 3.7 & 3.8 \\
\hline North Gosford Private & 5.9 & 5.6 & 5.3 & 4.9 & 4.9 & Other Area hospitals & 4.1 & 3.9 & 3.7 & 3.7 & 3.5 \\
\hline ALL HOSPITALS & 4.1 & 4.2 & 4.1 & 3.9 & 3.9 & ALL HOSPITALS & 3.7 & 3.6 & 3.6 & 3.4 & 3.4 \\
\hline \multicolumn{6}{|l|}{ Sydney West } & Greater Western & & & & & \\
\hline Auburn & 2.8 & 2.8 & 2.8 & 2.8 & 2.8 & Dubbo Base & 3.0 & 2.9 & 2.7 & 2.5 & 2.4 \\
\hline Blacktown & 3.1 & 3.0 & 3.0 & 3.0 & 2.9 & Mudgee & 3.5 & 3.2 & 3.2 & 2.9 & 3.2 \\
\hline Westmead & 3.3 & 3.4 & 3.3 & 3.2 & 3.2 & Bathurst Base & 3.3 & 3.4 & 3.2 & 3.1 & 3.1 \\
\hline The Hills Private & 5.6 & 5.5 & 5.2 & 5.0 & 4.8 & Orange Base & 3.1 & 3.4 & 3.2 & 3.2 & 3.3 \\
\hline Westmead Private & - & - & 4.8 & 4.9 & 4.7 & Broken Hill Base & 4.1 & 4.4 & 3.4 & 3.6 & 3.4 \\
\hline Blue Mountains & 3.7 & 3.5 & 3.5 & 3.7 & 3.6 & Other Area hospitals & 3.7 & 3.7 & 3.5 & 3.5 & 3.2 \\
\hline Nepean & 3.2 & 3.3 & 3.3 & 3.2 & 3.0 & ALL HOSPITALS & 3.3 & 3.3 & 3.1 & 3.0 & 3.0 \\
\hline Hawkesbury & 3.5 & 3.4 & 3.3 & 3.2 & 3.4 & Greater Southern & & & & & \\
\hline Nepean Private & - & - & 4.3 & 4.8 & 4.7 & Griffith Base & 3.4 & 3.1 & 3.1 & 3.1 & 3.1 \\
\hline Other Area hospitals & 5.1 & 4.9 & 4.3 & 4.0 & 3.1 & Wagga Wagga Base & 3.3 & 3.8 & 3.1 & 2.9 & 3.0 \\
\hline ALL HOSPITALS & 3.6 & 3.5 & 3.4 & 3.5 & 3.4 & Calvary, Wagga Wagga & 5.5 & 5.2 & 4.7 & 5.0 & 4.6 \\
\hline \multicolumn{6}{|l|}{ Hunter \& New England } & Goulburn Base & 3.3 & 3.5 & 3.5 & 3.4 & 3.4 \\
\hline Maitland & 3.2 & 3.4 & 2.9 & 2.6 & 2.5 & Queanbeyan & 3.4 & 3.4 & 3.2 & 3.1 & 3.2 \\
\hline Muswellbrook & 3.5 & 3.5 & 3.4 & 3.3 & 3.2 & Other Area hospitals & 4.0 & 3.9 & 3.6 & 3.7 & 3.6 \\
\hline Belmont & 3.5 & 3.6 & 3.1 & 3.3 & 3.1 & ALL HOSPITALS & 3.9 & 3.8 & 3.5 & 3.6 & 3.6 \\
\hline John Hunter & 3.9 & 3.6 & 3.3 & 3.3 & 3.2 & TOTAL NSW & 3.7 & 3.7 & 3.5 & 3.5 & 3.5 \\
\hline Christo Road Private & 5.5 & 5.3 & 4.9 & 4.9 & 4.7 & & & & & & \\
\hline Manning Base & 3.9 & 4.1 & 3.6 & 3.2 & 3.0 & & & & & & \\
\hline Armidale & 4.4 & 4.4 & 3.9 & 3.8 & 3.7 & & & & & & \\
\hline Inverell & 3.4 & 3.4 & 3.2 & 3.0 & 3.1 & & & & & & \\
\hline Moree & 4.0 & 3.7 & 3.6 & 3.3 & 3.5 & & & & & & \\
\hline Tamworth Base & 3.6 & 3.8 & 3.6 & 3.5 & 3.3 & & & & & & \\
\hline Other Area hospitals & 4.2 & 3.9 & 3.8 & 4.0 & 3.8 & & & & & & \\
\hline ALL HOSPITALS & 3.9 & 3.8 & 3.5 & 3.5 & 3.3 & & & & & & \\
\hline
\end{tabular}

Source: Linked data of the NSW Midwives Data Collection and NSW Inpatient Statistics Collection (HOIST). Centre for Epidemiology and Research, NSW Department of Health.

\# Hospitals with more than 200 deliveries are identified individually. All hospitals include all public and private hospitals. 


\section{Indicators of obstetric care}

The Australian Council on Healthcare Standards and the Royal Australian and New Zealand College of Obstetricians and Gynaecologists have endorsed seven clinical indicators for use in Hospitals.
Table 124 shows aggregate information for these indicators for all NSW hospitals and comparative information for all participating hospitals in Australia.

\section{TABLE 124}

\section{CLINICAL INDICATORS FOR OBSTETRICS, NSW AND AUSTRALIA, 2003}

Indicator description

NSW

$\%$

$\begin{array}{cc}\text { Australia } \\ \text { 20th } & \text { 80th } \\ \text { Centile } & \text { Centile }\end{array}$

Indicator 1:Induction of labour other than for defined indications

1.1 The number of patients undergoing induction of labour other than for defined indications \# (excluding augmentation of labour) as a percentage of the total number of patients undergoing induction of labour for any reason (excluding augmentation of labour).

1.2 The number of patients undergoing induction of labour other than for defined indications \# (excluding augmentation of labour) as a percentage of the total number of patients delivering (excluding augmentation of labour).

Indicator 2:The rate of vaginal delivery following primary caesarean section.

2.1 The number of patients delivering vaginally following previous primary caesarean section as a percentage of the total number of patients delivering who have had a previous primary caesarean section with no intervening pregnancies greater than 20 weeks gestation.

Indicator 3:Primary caesarean section for failure to progress.

3.1 The number of patients undergoing primary caesarean section for failure to progress after a period of labour with cervical dilation of $3 \mathrm{~cm}$ or less as a percentage of the total number of patients undergoing primary non-elective caesarean section.

3.2 The number of patients undergoing primary caesarean section for failure to progress after a period of labour with cervical dilation of more than $3 \mathrm{~cm}$ as a percentage of the total number of patients undergoing primary non-elective caesarean section.

Indicator 4:Primary caesarean section for fetal distress.

4.1 The number of patients undergoing primary caesarean section for fetal distress as a percentage of the total number of patients delivering.

4.2 The number of patients undergoing primary caesarean section for fetal distress as a percentage of the total number of patients delivering by primary caesarean section.

Indicator 5:Incidence of intact lower genital tract in primiparous patients delivering vaginally.

5.1 The number of primiparous patients not requiring surgical repair of the lower genital tract as a percentage of the total number of primiparous patients delivering vaginally.

Indicator 6:Apgar scores.

6.1 The number of babies born with an Apgar score of four or below at five minutes post delivery as a percentage of the total number of babies born.

6.2 The number of babies born with an Apgar score of six or below at ten minutes post delivery as a percentage of the total number of babies born. ${ }^{\# \#}$

Indicator 7:Term infants transferred or admitted to a neonatal intensive care unit for reasons other than congenital abnormalities.\#\#\#

7.1 The number of term babies transferred/admitted to a neonatal intensive care unit for reasons other than congenital abnormality as a percentage of all term live babies born.

Source: NSW Midwives Data Collection (HOIST). Centre for Epidemiology and Research, NSW Department of Health. The Australian Council on Healthcare Standards. Determining the Potential to Improve Quality of Care. 5th Edition, ACHS Clinical Indicator Results for Australia and New Zealand 1998-2003. Sydney: The Australian Council on Healthcare Standards, 2004.

\# Defined indications include: diabetes, hypertensive disease, fetal distress, fetal death, chorioamnionitis, blood group isoimmunisation, prelabour rupture of membranes, prolonged pregnancy (41 or more weeks), and suspected intrauterine growth retardation.

\#\# NSW data not collected.

\#\#\# NSW data are provided by hospital of birth and may be under-enumerated. Infants transferred to another hospital and then admitted to NICU for reasons other than congenital abnormality may not be reported by the hospital of birth. 